\title{
$(20, y-910318-16$
}

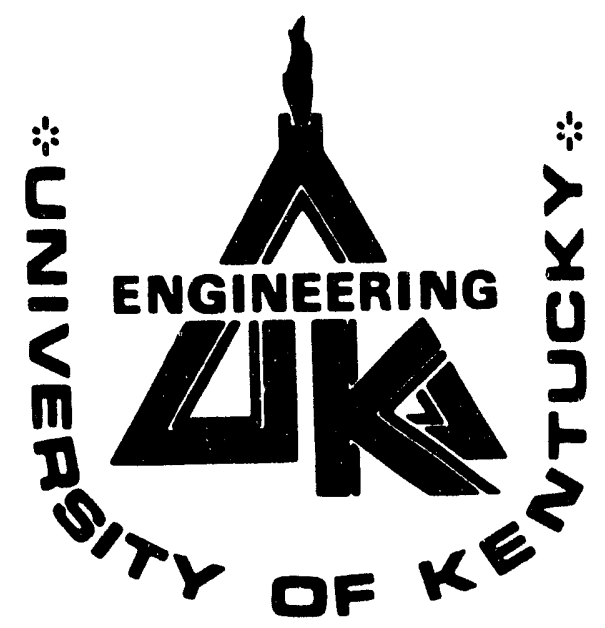

\section{DETERMINATION OF LOCAL RADLTTVE PROPERTIES IN COAL-FIRED FLAMES}

(Grant No: DE-FG22-87PC70916)

TECHNICAL PROGRESS REPORT - DOE/PC/79916-0

THIRD QUARTER OF THE THIRD YEAR : $9 / 15 / 1990 \cdot 6 / 15 / 1990$

Subrnitled to the

U.S. Department of Energy

Pitloburgh Energy Teehnology Center

M. Pinar Mengüc

Department of Mechanical Engineering

College of Engineering

University of Kentucky

Lexington, Kentucky 40506-0046 


\title{
DETERMINATION OF THE RADIATIVE PROF ERTIES OF PULVERIZED-COAL PARTICLES
}

(Grant No: DE-FG22-87PC79916)

\author{
TECHNICAL PROGRESS REPORT - DOE/PC/79916-9
}

THIRD QUARTER OF THE THIRD YEAR

MARCH 15, 1990 - JUNE 15, 1990

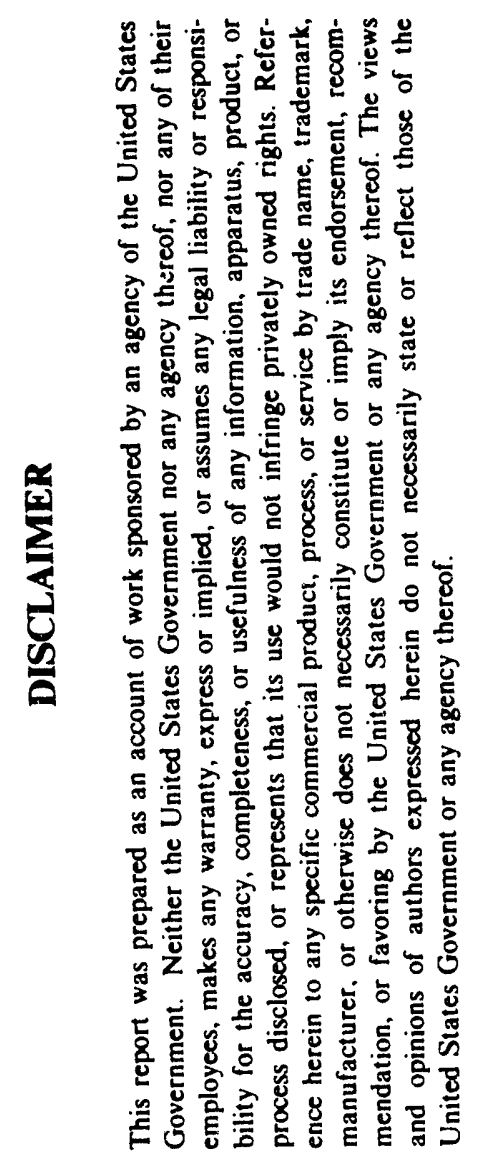

\author{
Submitted to the \\ U.S. Department of Energy \\ Pittsburgh Energy Technology Center \\ by \\ M. Pinar Menguc (Associate Professor) \\ D. Dsa (Graduate Student) \\ S. Manickavasagam (Graduate Student) \\ P. Dutta (Graduate Student) \\ A. Mahadeviah (Graduate Student) \\ Department of Mechanical Engineering \\ University of Kentucky \\ Lexington, $K Y$ 10506-0046
}

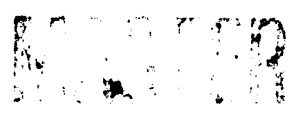

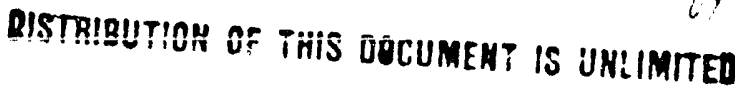




\section{PREFACE}

This report summarizes the progress on the ongoing research effort for determining the radiative properties of coal particles, which is funded by a grant from the U.S. Department of Energy Pittsburgh Energy Technology Center (Grant No: DE-FG22-87PC79916). The period covered in this report is the third quarter of the third year.

During this period, we conducted experiments using both the $\mathrm{CO}_{2}$-laser nephelometer as well as Mercury-arc lamp-monochromator system. The data obtained from the transmission and scattering measurements were reduced using an analytical/numerical inverse radiation analysis. The experiments were performed for only Blind Canyon coal. Preliminary results are discussed in this report. Note that an extended version of this work is submitted for presentation at the ASME/JSME Joint Thermal Engineering Conference, which will be held at Reno, Nevada in March 1991.

Within the next quarter, we will make more measurements with different coal types. Also, we will start the flame experiments. Currently, we are manufacturing a new burner which will allow us to monitor coal particle temperature very accurately. 


\section{Contents}

1 INTRODUCTION 1

1.1 Background .......................... 1

1.2 Optical and Radiative Properties . . . . . . . . . . . . . . 2

1.3 Objectives ........................ 3

2 ANALY'TICAL FORMULATION 3

2.1 Analysis of Multiple Scal tering by a Particle Cloud . . . . . . . . . . . 4

3 EXPERIMENTAL SYSTEM 6

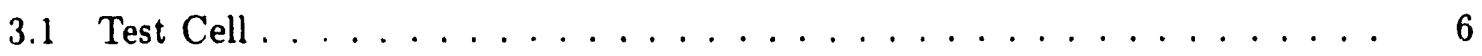

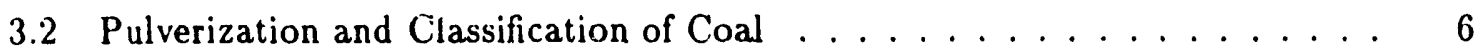

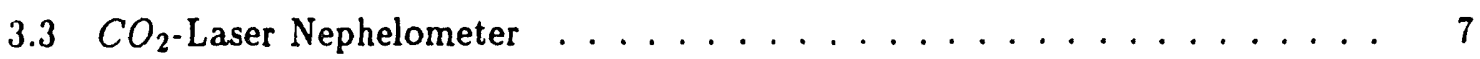

3.4 Mercury Arc Lamp-Monochromator System . . . . . . . . . . . . . . 9

3.4 .1 Fibre Optic Probes . . . . . . . . . . . . . . . . 10

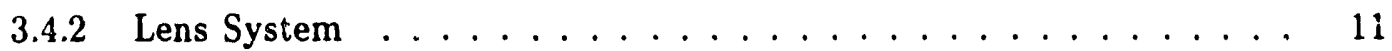

3.4 .3 Photomultiplier Tubes . . . . . . . . . . . . . . 11

3.4 .4 Data Acquisition Set-Up . . . . . . . . . . . . . . 11

4 EXPERIMENTS AND DISCUSSION OF RESULTS 12

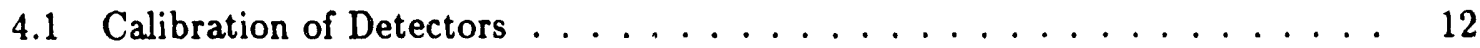

4.2 Coal Experiments . . . . . . . . . . . . . . . . . . 13

4.2.1 Scattering/Transmission Experiments at $10.6 \mu \mathrm{m} \ldots \ldots \ldots 13$

4.2 .2 Inverse Radiation Analysis . . . . . . . . . . . . . . 14

4.2.3 Transmission Measurements at Visible Wavelengths . . . . . . . 16

5 CONCLUSIONS AND RECOMMENDATIONS 18 


\title{
Determining the Radiative Properties of Pulverized-Coal Particles from Experiments
}

\author{
M. P. MENGÜÇ, D. D'SA, and S. MANICKAVASAGAM \\ Department of Mechanical Engineering, University of Kentucky, Lexington, KY 40506
}

\begin{abstract}
For accurate modeling of radiative transfer in combustion systems, radiative properties of combustion products are required. It is usually difficult to calculate the properties of nonhomogeneous and irregular-shaped pulverized-coal and char particles, because of the lack of information on optical constants and unavailability of simple and accurate theoretical models. Because of this, it is preferable to determine the required properties from experiments in situ. This can be accomplished by combining optical diagnostic techniques with inverse analyses of radiative transfer problem.

In this study, experiments were conducted using a $\mathrm{CO}_{2}$-laser nephelometer to measure angular distribution of light scattered by a cold-layer of pulverized-coal particles. The data obtained from the experiments were used along with a new step-phase function approximation in a numerical inverse radiation scheme to obtain "effective" extinction coefficient and scattering phase function for coal particles in narrow size distributions. In addition to that, a mercury-arc-lamp monochromator system was used to obtain spectral absorption coefficient of coal particles as a function of wavelength and coal size.
\end{abstract}

(SUbmitTed for presentation at the ASME/JSME Joint Thermal Engineering Conference, Reno, Nevada, in March 1991.) 


\section{INTRODUCTION}

\subsection{Background}

Radiation is the most significant mode of heat transfer in combustion systems like furnaces, diffusion and pulverized coal flames (Blokh, 1984; Viskanta and Mengüç, 1987). The contribution of radiation to the total heat transfer increases as the size of the system increases. For example, in large-scale pulverized-coal fired furnaces, it may account for as much as $90 \%$ of the total heat transfer. In such furnaces, the radiated energy propagates from the flame to the heat absorbing surfaces through a participating, i.e. emitting, absorbing and anisotropically scattering medium which is composed of pulverized-coal, char, fly ash, soot, and gaseous products of combustion.

In order to design or model combustion systems, the accurate solution of the radiative transfer equation is essential. This can be accomplished only if the radiative properties of the combustion products, especially those of the particulate matter is known. Unfortunately, this informatior. is usually not available.

Given the size parameter $(x=\pi D / \lambda)$ and the complex index of refraction of a particle, the required radiative properties such as the absorption, scattering and extinction coefficients can be obtained from the Lorenz-Mie theory (Van de Hulst, 1957; Kerker, 1969; Bohren and Huffman, 1983). The theory, however, provides only a first approximation to the problem of scattering by non-spherical particles. Recently, Mugnai and Wiscombe (1989) have indicated that using a single effective diameter yields wroneous results as sphericity is more the exception than the rule in nature. Considering that most of the particles of interest such as pulverized coal and char are non-homogeneous and non-uniform in shape, the use of the Lorenz-Mie theory to predict their radiative properties is questionable. To be able to understand on a micro-scale, physical phenomena such as radiation-combustion interactions and the effects of pores and inhomogeneities on the scattering and absorption of incident radiant energy, the effect of irregularly shaped particles should be considered.

Several different methodologies have been reported in the literature to determine the radiative properties for irregularly shaped nonhomogeneous particles, An extensive review of the available approaches was given by Wiscombe and Mugnai (1986). These techniques are classified as integral and differential methods. The Extended Boundary Condition method (EBCM; also known as the T-Matrix method) is the most versatile and powerful of the integral methods. First introduced by Waterman in 1965, it was subsequently improved and adapted for computer applications (Barber and Yeh, 1975; Wiscombe and Mugnai, 1986). On the other hand, differential techniques are more straightforward to model compared 
to the integral approaches (Yeh and Mei, 1980). However, convergence of the solution is more difficult to achieve because of the difficulties associated with matching the boundary condition on the surface of the obstacle.

Another technique, which differs from the two main methods mentioned above was given by Purcell and Peunypacker (1973). In this approach, the obstacle can be considered as being composed of several dipoles, so that the incident electromagnetic wave induces a dipole moment in each region. This analysis is particularly useful for studying light scattering by particles with iahomogeneous optical properties and can be employed for size parameters ranging to abou 4 for weakly absorbing particles. In general, all of these schemes have certain drawbacks and limitations, and none of them can be applied universally, especially to coal/char clouds where the particle shape is completely arbitrary.

\subsection{Optical and Radiative Properties}

An accurate determination of radiative properties involves a thorough knowledge of the complex index of refraction, volume fraction, shape and size distribution of the particles involved. Of these, the most fundamental is the spectral complex index of refraction, ( $\tilde{n}_{\lambda}=n_{\lambda}-i k_{\lambda}$ ), which is a wavelength dependent property. Variation of the complex index of refraction of particles in the near-infrared region of the spectrum is required as it is in this region that the flame strongly emits radiation. Using the information on particle refractive index, the spectral absorption and scattering coefficients along with scattering phase function for the particle of interest can be obtained, provided the shape is known.

Data for a wide range of coals using Fresnel reflectance techniques have been listed by Stull and Plass (1960), Field et al. (1967), Foster and Howarth (1968), Blokh and Burak (1973). Stull and Plass studied the coal within the infrared region of the spectrum up to 6 $\mu \mathrm{m}$, while Foster and Howarth obtained data for coal up to a wavelength of $10 \mu \mathrm{m}$. Blokh (1984) listed the relations used to determine the refractive index for different wavelengths in the region 0.5 to $4 \mu \mathrm{m}$ for char and various types of USSR coals. It is observed that the carbon content of coal affects the refractive index, both real and imaginary parts. Brewster and Kunitomo (1984) using an ex situ particle extinction technique have also reported complex index of refraction data for coals. All other techniques are based on the recovery of the index of refraction of particles from experiments performed on one-dimensional slabs using a Fresnel-reflection technique applicable to smooth surfaces. However, it is difflcult to obtain polished surfaces (roughness less than $\lambda / 4$ ), and the accuracy of the coefficients so recovered is questionable.

The particle extinction technique is more accurate than the others; however, because the 
particle shape has to be assumed to recover the corresponding complex index of refraction, it is not immune to physical errors in addition to those from experiments. It is also worth to note that the refractive index data given in these studies assume coal as homogeneous. This is not true especially during the pyrolysis of coa'. Even if accurate refractive index data are available, reliable prediction of the properties of coal may not be possible because of the uncertainities in the particle shape. Given this, it is preferable to perform experiments which yield "effective properties" as a function of coal rank: particular size distribution, temperature, and combustion characteristics.

In situ measurements of coal particle properties have not been conducted exterisively. Grosshandler and Monteiro (1981) studied the effective emissivity of pulverized coal flames and gave possible values of complex index of refraction that would yield the effective emissivities they were able to measure. Solomon et al. (1986) measured the transmissivity and emissivity of laboratory scale pulverized-coal flames. However, so far no extensive work on the determination of absorption and scattering coefficients and the scattering phase function of pulverized coal particles from experiments has been reported in the literature.

\subsection{Objectives}

Our objective in this study is to report an experimental/theoretical methodology to determine the "effective" radiative properties of coal particles as a function of narrow size distributions. For this purpose, a $\mathrm{CO}_{2}$-laser nephelometer and a Mercury-arc lamp and monochromator system are used. The results are reported for a subbituminous coal (Blind Canyon, Utah). An inverse radiation analysis is followed to reduce the experimental data to required radiative properties.

\section{ANALYTICAL FORMULATION}

In this section, an analytical formulation for the forward problem will be summarized to determine transmission and scattering of a laser beam incident on a cloud of particles. This formulation is later employed in the inverse radiation analysis to predict the effective radiative properties of the cloud. If these properties are then used in the direct formulation, the transmitted and scattered intensity distribution obtained outside the cloud should match the experimentally measured flux and intensity values.

In the experiments, the laser radiation transmitted and scattered by the particles are measured. The angular distribution of the scattered radiation intensity is dependent on the scattering phase function and system geometry. The scattering phase function, also referred 
to as the scattering diagram, $\Phi_{\lambda}\left(\overrightarrow{s^{\prime}} \rightarrow \vec{s}\right)$, represents the probability of the radiation propagating in the given direction $\overrightarrow{s^{\prime}}$, being scattered by the particles in its path into a different direction $\vec{s}$. It can be given as a series expression of Legendre polynomials as:

$$
\Phi_{\lambda}(\Psi)=\sum_{n=0}^{N} a_{n, \lambda} P_{n}(\Psi)
$$

where $a_{n, \lambda}$ are the expansion coefficients and are defined by:

$$
a_{n, \lambda}=\frac{1}{2 n+1} \int_{\Omega=4 \pi} \Phi_{\lambda}(\Psi) P_{n}(\Psi) d \Omega
$$

Here, $P_{n}$, are the Legendre polynomials of degree $n$.

The form of the phase function depends to a great extent on the size parameter $x$. For $x \ll$ 1 , the phase function remains almost the same even when $x$ changes and only the overall quantity of energy scattered by the particle in all the directions undergoes a change. For $x \gg 1$, most of the radiation is concentrated in the direction of propagation of the incident beam. For intermediate values of $x$, the Lorenz-Mie theory is to be used to determine the scattering phase function if the particles are spherical. For irregularly shaped particles, the phase function can be determined from experiments.

\subsection{Analysis of Multiple Scattering by a Particle Cloud}

Laser radiation incident on a cloud of particles is absorbed and scattered before it leaves the medium. A forward analysis can be presented to determine the effect of particle radiation properties and medium geometry on the intensity distribution outside the cloud. If the detectors with small but finite solid angles are used in the experiments, then the recorded values are not intensities but radiosities. Here, radiosity is defined as the intensity in tegrated over a small detector solid angle.

Figure 1 depicts a cylindrical medium, center $O$ and diameter $d$, in which is present a cloud of absorbing and scattering particles. As before, radiation of intensity $I_{0}$ and wavelength $\lambda$ is incident on the medium. A detector of area $A_{d}$ is positioned at an angle $\theta$ and at a distance $z$ from the origin. The solid angle subtended by the detector is $\Delta \Omega$. The cross-section of the area seen by the detector is assumed to be constant throughout the test-cell. Also, the properties of the particle cloud remain the same in the solid angle $\Delta \Omega . P_{1}$ and $P_{2}$ represent the intersection of the extremities of the solid angle and the direction of propagation of the beam.

The beam travels to the point $P_{1}$, distance $l_{1}$ from $A$. The beam could be scattered from anywhere along the path $\overline{P_{1} P_{2}}\left(=L_{2}\right)$. As only radiation reaching the detector is of interest, 
only those angles that cause the radiation to fall on the detector are considered. The beam exits at the point $C$ and is only attenuated along the path $\overline{B C}$ where $B$ is any point between $P_{1}$ and $P_{2}$. Intensity at $P_{1}$ due to the attenuation along the path $\overline{A P_{1}}$ is given by:

$$
I_{P_{1}}=I_{0} e^{-\bar{\beta} l_{1}}
$$

where $\bar{\beta}$ is the effective extinction coefficient for the medium as defined by

$$
\bar{\beta}=\kappa+\sigma\left(1-\frac{\omega(0) \Delta \Omega}{4 \pi}\right)
$$

Following the analysis given by Agarwal and Mengüç (1990), the intensity of the beam incident on the detector due to first-order scattering is given as

$$
I_{D, 1}(\theta)=\int_{0}^{L_{2}} \sigma \cdot \frac{\Phi(\theta) \cdot \Delta \Omega}{4 \pi} I_{0} e^{\left(-\bar{\beta}\left[l_{1}+l_{2}+l_{3}\right]\right)} d l_{2}
$$

where the angle $\theta$ is a function of the distance $l_{2}$. Equation (4) can be put into a form suitable for numerical solution using Gaussian quadrature techniques.

If the number density of the particles in the medium increases, higher orders of scattering have to be taken into account. The analysis presented in the earlier section can be extended to two orders of scattering. Following Agarwal and Mengüç (1990), the second-order scattering contribution of radiation incident on the detector from all the particles present in the entire test-cell area can be written as :

$$
I_{D, 2}=I_{0} \int_{0}^{L_{1}} \int_{0}^{L_{3}}\left(\frac{\sigma}{4 \pi}\right)^{2} \Phi\left(\theta_{1}\right) \cdot \Phi\left(\theta_{2}\right) \cdot e^{-\bar{\beta}\left(l_{1}+l_{2}+l_{3}\right)} \cdot d l_{3} \cdot d l_{1}
$$

where $d l_{1}$ is an elemental length of the path $\overline{P_{1} P_{2}}\left(=L_{1}\right)$ and $d l_{3}$ is the elemental length of the path $C C^{\prime}$ (see Figure 2).

The total power of the radiation incident on the detector from second order scattering expressed in terms of the angular radiosity $E_{D}\left(=\left(I_{D, 1}+I_{D, 2}\right) \cdot \Delta \Omega\right)$ is given as $P_{D}=$ $E_{D} \cdot \Delta A$, where $\Delta A$ is the cross-sectional area of the laser beam. The analysis here assumes that $\Delta A$ is small. For large $\Delta A$, a more rigorous analytical formulation is necessary. Such a formulation was recently obtained by Mengüç and Manickavasagam (1990).

For a medium of optical thickness $\tau<0.2$, the contribution of first order is sufficient to predict the scattered intensity as a function of angle. For $0.2<\tau<0.5$, two orders of scattering have to be included for accurate prediction of scattered intensity (Agarwal and Mengüç, (1990)). They have also shown that for optical thicknesses greater than 1.0 even higher orders of scattering must be included. 


\section{EXPERIMENTAL SYSTEM}

\subsection{Test Cell}

To obtain a one dimensional flow of coal particles two test-cells were designed: a planar one and a cylindrical one. The cells consist of two sections: the upper portion of cylindrical crosssection and the lower portion of either a trapezoidal cross-section narrowing throughout its length, or a cylindrical cross-section. The test-cell with a circular cross-section gave a very smooth, homogeneous flow of particles (see Figure 3). It consists of a Plexiglas frustrum with an exit diameter of $12.5 \mathrm{~mm}$. Connected to this is a stainless steel pipe, I.D. 9.5 $\mathrm{mm}$ and O.D. $12.5 \mathrm{~mm}$. The particles exiting the pipe form an even, homogeneous flow. Pulverized coal particles are fed to the test-cell from a fluidized-bed feeder through an offtake tube. Coal from the feeder issues out into the cylindrical portion of the test-cell as a fountain through a concentric jet. The weight of the feeder is monitored using a load cell and a strip chart recorder.

The fluidized-bed was designed by Altenkirch et al. (1978). The original system performed well when the coal particles of diameter $38 \mu \mathrm{m}$ or larger were used. For particles of smaller diameter the flow tended to be erratic and uneven the cause of which was determined to be the clogging of the off-take tube because of the uneveness in the flow. A new off-take tube was used to obtain a much steadier flow even with a size distribution of $0.25 \mu \mathrm{m}$. It also enabled us to obtain much larger flow rates than was possible before.

\subsection{Pulverization and Classification of Coal}

In the experiments, a subbituminous coal (Blind Canyon, Utah; from Pennsylvania State University) was used. First, large millimetre sized coal particles were mixed with an anticaking agent (Aerosil R972) and pulverized to fine size in a pulverizer (Alpine Laboratories Universal Mill, Model 100LU). The micron sized particles were then passed through a ZigZag classifier (Alpine Laboratories, Model $4 \mathrm{WM}$ ) to cut off particles greater than $75 \mu \mathrm{m}$. Initially, only the Zig-Zag classifier was employed. However, this method of classification did not give the desired range of size distributions as observed from electron microscope photographs. Therefore, a shaker (Gilson Co. Inc., Model SS-8R), and six sieves \# 230 (63 $\mu \mathrm{m})$, \# $270(53 \mu \mathrm{m})$, \# $325(45 \mu \mathrm{m})$, \# $400(38 \mu \mathrm{m})$, \# $450(32 \mu \mathrm{m})$, \# $500(25 \mu \mathrm{m})$ (Gilson Co. Inc., Models V8BF) were used to obtain narrow size distributions within the ranges of 0-25 $\mu \mathrm{m}, 25-32 \mu \mathrm{m}, 32-38 \mu \mathrm{m}, 38-45 \mu \mathrm{m}, 45-53 \mu \mathrm{m}$, and 53-64 $\mu \mathrm{m}$. The smallest size range was collected in a pan. Each classification run was carried out with approximately $75 \mathrm{gm}$ of coal and shaken for ten minutes. This ensured enough time for all possible particles to pass 
through the individual sieves. Once the run was finished each size distribution was stored in individually marked airtight plastic bottles to prevent oxidation of coal. The particles that remained in the uppermost sieve were pulverized and classified again by the same procedure detailed above. The entire operation was carried out taking care to prevent contamination or alteration of the particles in any manner.

\section{3 $\mathrm{CO}_{2}$-Laser Nephelometer}

A polar nephelometer is a device that can detect and measure light scattered at different angles (Bohren and Huffman, 1983). It consists of a collimated light source incident or: a scattering cell. A system of detectors is used to collect the scattered light by mounting them on an arm that rotates about the scattering cell. Resolution and sensitivity of the instrument are mutually dependent, usually one at the expense of the other. The optimum is achieved when the incident beam is the same size at the scattering volume as at the detector. The intersection of the beam with the test-cell area and the detector field of view determines the effective scattering volume from which the measurements are made. This volume is dependent on the angle made by the detector with the scattering cell. A recent review of the different nephelometers employed by various researchers and their techniques of measurement is given by Agarwal and Mengüç (1990).

A CO 2 -laser (Coherent General, Model M42, 50 watts) is employed in these experiments to produce a collimated, intense beam of high power which allows for detection of scattered light for a wide range of angles. The laser itself is mounted on an optical breadboard which in turn is mounted on a service cart. A silver mirror (II-VI Inc., 2208-1000CT) is used to direct the beam through an angle of $90^{\circ}$ and thus incident on the test cell area. For a laser current of $14 \mu \mathrm{A}$, the maximum laser output was obtained at a frequency of $33.5 \mathrm{~Hz}$.; all the results reported in this study were obtained at this pulser frequency.

The beam emitted directly by the laser was circular in shape as well as collimated throughout its length. This was tested by making burns on both thermally-sensitive and ordinary paper; from which the diameter of the beam was determined to be $6 \mathrm{~mm}$. This is very close to the manufacturers specifications listed for the gas flows and currents used.

The $\mathrm{CO}_{2}$-laser nephelometer used in this study consists of a $\mathrm{LiTaO}_{3}$ crystal detector (Hamamatsu P2613) mounted on an optical rail which in turn is mounted on a rotary base. The base can be rotated to take both transmission and scattering readings. Also mounted on the optical rail is an aperture to limit the the solid angle of the radiation incident on the detector.

The output of the detector is fed to a lock-in amplifier (Princeton Applied Research Model 
128) via a multi-channel $\mathrm{LiTaO}_{3}$ buffer, which serves to standardize the impedance of the entire detector-data acquisition circuit. The lock-in filters out noise from the detector output and the signal so produced is preserved both as an analog signal on a tape-deck (Kyowa-Dengyo RTP-600) and in digital format on a computer (IBM PC-XT). Routines are available that store the data in files and also convert it so that it can be viewed on the terminal screen as a graph.

Originally, the $\mathrm{CO}_{2}$-laser beam was aligned using a Helium-Neon laser (Bush, 1989). After enough experience was gained, however, it was possible to make finer adjustments of the beam using the detectors alone Therefore, in this phase of the experiments, the Heliumveon laser was not used.

The power of the laser beam is monitored regularly using a second detector, which also allowed us to normalize transmission and scattering readings.

To limit the solid angle seen by the detector, two different apertures were used for scattering and transmission. The solid angle $\Delta \Omega$, subtended at the detector and the coal cloud is determined as (see Figure 5):

$$
\Delta \Omega=\frac{\pi d_{d}^{2}}{4\left(l_{1}-l\right)^{2}}=\frac{\pi d_{a p}^{2}}{4 l^{2}}=\frac{\pi d_{f l}^{2}}{4\left(l_{2}+l\right)^{2}}
$$

The distance $l$ can be obtained from the first two terms of Equation (6) as:

$$
\frac{d_{d}}{\left(l_{1}-l\right)}=\frac{d_{a p}}{l}
$$

Therefore,

$$
l=\frac{d_{a p} l_{1}}{\left(d_{d}+d_{a p}\right)}
$$

Knowing $l$, the solid angle $\Delta \Omega$ can be determined from Equation (6). The effective diameter of the area from which the radiation is incident on the detector is then determined from the last term of Equations (6) as:

$$
d_{f l}=2\left(l_{2}+l\right) \sqrt{\frac{\Delta \Omega}{\pi}}
$$

Apertures for transmission and scaitering measurements are detailed below:

- For transmission: As the laser power is the strongest at the forward angle $\left(0^{\circ}\right)$, the detector should be exposed to as small a portion of the beam as possible to prevent its burning up. The mounts for the detector and aperture are kept fixed to avoid 
unnecessary errors, so the only option is to employ a small diameter aperture. An aluminium disc with an opening of $0.61 \mathrm{~mm}$ at its centre is used; the solid angle so subtended is 0.00057 steradians and the effective diameter of the area "seen" by the detector is $3.8 \mathrm{~mm}$, as determined from Equations (6) and (9). To eliminate the possibility of radiation penetrating the aluminum, a similar disc made of Plexiglas with an aperture of $1 \mathrm{~mm}$ is used behind the aluminium disc as backing. At the same time to keep reflections off the aperture wall to a minimum, the thickness of the aluminum did not exceed $0.8 \mathrm{~mm}$.

- For scattering: As the radiation scattered is at least three orders of magnitude smaller than transmission, the solid angle subtended by the detector has to be increased to prevent a great degree of noise from affecting the accuracy of the measurements. Yet, the two solid angles should be of comparable magnitude. An identical aperture as before but with a diameter of $1.7 \mathrm{~mm}$ is used. The solid angle subtended by the detector using this aperture is 0.0016 steradians while the effective diameter of the area from which radiation is incident on the detector is $7.1 \mathrm{~mm}$.

In either case diffraction effects are negligible as the wavelength $(\lambda=10.6 \mu \mathrm{m})$ is much smaller than the diameter of the apertures $\left(\sim 10^{3} \mu \mathrm{m}\right)$.

\subsection{Mercury Arc Lamp-Monochromator System}

The results obtained from the $\mathrm{CO}_{2}$-laser experiments are restricted to the wavelength of $10.6 \mu \mathrm{m}$. It is desirable to perform the experiments at visible and near infra-red wavelengths as this range of the spectrum is important in flames. For this purpose, a Mercury Arc Lamp (Oriel Corp., Model 6285-500W-Hg) is used as a source and a Monochromator (Oriel Corp., Model 77250) is employed to perform experiments one wavelength at a time.

The monochromator is an instrument that can isolate and transmit a narrow band of spectral radiation from a broad range of wavelengths. It is capable of being used not only for visible wavelengths but also for ultra-violet and infra-red regions of the spectrum as well. There are two types of monochromators namely the filter monochromator and the grating monochromator. The latter is opted for in these experiments because it provides a wider spectral range and is the more precise of the two.

The grating used with this system is of the blazed type (Oriel Corp., Model 77299) which is a ruled grating with 600 lines per $\mathrm{mm}$. The wavelength for which the grating is most efficient is $1.0 \mu \mathrm{m}$, i.e. the greatest fraction of the incident light is diffracted at $1.0 \mu \mathrm{m}$ wavelength. It is, however, useful throughout the region $0.67-1.5 \mu \mathrm{m}$. 
A Mercury Arc lamp (Oriel Corp., Model $6285-500 \mathrm{~W}-\mathrm{Hg}$ ) is used in the experiments, as its output spectrum is dominated by strong mercury lines in this spectral range. The lamp is rated for 500 Watts with a luminous flux of 30,000 lumens and an average brightness of 300 candela $\mathrm{mm}^{-2}$. The lamp is mounted in a Universal Arc Lamp Housing (Oriel Corp., Model 66011), and used with corresponding power-supply source (Oriel Corp., Model 8530).

A variable slit assembly (Oriel Corp., Model 77263) is employed in the experiments, which is continously adjustable to widths from $20 \mu \mathrm{m}$ to $3.2 \mathrm{~mm}$ and to heights from $1 \mathrm{~mm}$ to $12 \mathrm{~mm}$. To optimize the monochromator effectiveness, slits of the correct height and width have to be used.

In order to detect only the radiation enitted by the source, but not that from the surroundings, the incident radiation is to be modulated. This is achieved using an Optical Chopper (Stanford Research Systems, Model SR540) which modulates the input light beam into a square wave with a variable frequency that can be accurately determined with the help of a micrometer frequency control dial. The frequency to be used is determined once the entire data acquisition apparatus is set up. The frequency is varied till the maximum output is obtained as determined from the data acquisition set up. The results reported here were obtained at a frequency of $400 \mathrm{~Hz}$.

\subsubsection{Fibre Optic Probes}

To bring the radiation output from the monochromator arc-lamp system onto the test-cell area, a fibre-optic cable is employed. The advantages of optical fibres are well known, but the main reason for employing one in this experiment is because it eliminates the need for setting up the Mercury arc lamp-monochromator assembly precisely near the test-cell.

The fibre used is a large core optical fibre (Oriel Corp., Model 77513). This has a core diameter of $800 \mu \mathrm{m}$ with a cladding thickness of $100 \mu \mathrm{m}$. The core, made of UV grade silica, is surrounded by silica glass cladding with an outer jacket of black nylon. The core has a refractive index of 1.452 at $850 \mathrm{~nm}$ while for the cladding it is 1.438 at $850 \mathrm{~nm}$. Its numerical aperture (NA) is $0.22 \pm 0.02$. For a length of $1 \mathrm{~m}$, the output from the fibre retains the characteristics of the beam launched into the fibre. The ends of the fibre are fitted with SMA connectors which allow for easy coupling to the monochromator.

The output from the monochromator can be directly input into a fibre. This is done using a Monochromator Adapter (Oriel Corp., Model 77852). This ensures that there is negligible leakage of radiation in transferring light from the monochromator to the fibre nor can stray light enter the fibre in any way.

To bring the fibre in close proximity with the test-cell, it is mounted on an optical rail 
using a Single-Fibre-Holder (Oriel Corp., Model 77848). This ensures the steadiness of the fibre in all three axes.

\subsubsection{Lens System}

The beam exiting the optic fibre is a diverging one. To be able to pass this beam through the pulverized-coal particle cloud, a system of lenses has to be designed. A diagram of the optical system used, is depicted in Figure 6. Here, $L 1$ (Newport Corp., Model KBX043) is a biconvex lens of focal length $19 \mathrm{~mm}, L 2$ (Newport Corp., Model KBX046) is also a biconvex lens but with a focal length of $25.4 \mathrm{~mm}$. These two lenses serves to convert the diverging beam from the exit of the fibre into a collimated beam. Collectic $\Omega$ of the transmitted radiation is accomplished using a plano-isnvex lens $L 3$ (Newport Corp., Model KPX076) of focal length $25.4 \mathrm{~mm}$. This focusses the transmitted beam onto the fibre optic entrance, completely filling its acceptance angle.

\subsubsection{Photomultiplier Tubes}

In the experimental system, a side-window reflection mode type photomultiplier tube (PMT) ( $R C A$, Model 4840) with multialkali photocathodes is used. To prevent stray light from affecting the performance of the PMT, a Plexiglas housing was designed and manufactured in house. This was painted with a highly absorbing, diffusely reflecting ultra flat black paint (Krylon \# 1602), to isolate the interior. An opening was provided in the wall for the optic fibre. The base of the PMT ( $R C A$ Model PF1041P) is mounted on the inside of the box in such a way as to allow the optic fibre to be beamed directly onto the photocathode. To ensure that the fibre optic is stationary a special holder is designed and manufactured in house. This meshes with a dove tail optical rail mounted in the box. The base of the holder is fastened using a pair of set screws. To prevent stray electromagnetic radiation affecting the PMT, it is covered with a magnetic shield.

\subsubsection{Data Acquisition Set-Up}

The output from the PMT is further amplified using an in-house built power source/amplifier, and connected to a a lock-in amplifier (Stanford Research Systems, Model SR510). The output of the lock-in amplifier is in analog form. It is converted to a digital signal and stored in a computer via an A/D board (Data Translation, DT2828).

Data is recorded in and displayed on a computer screen (IBM PC/AT) using available routines (Labtech Notebook). This allows for simultaneous recording of data and calculation 
of variance of the data. Sampling of data can be varied but is kept constant at a rate of 10 $\mathrm{Hz}$ every 15 seconds. Thus a recorded/displayed point is an average of 150 data points.

\section{EXPERIMENTS AND DISCUSSION OF RESULTS}

Two different types of experiments are conducted, one with the $\mathrm{CO}_{2}$-laser nephelometer and the other with the Mercury-Arc Lamp Monochromator system. In this section, the experiments and the procedures followed to interpret the raw data are discussed. First, the calibration of the detectors of the nephelometer using the glass particles is given. This is followed by a discussion of the experiments performed with the different size distributions of pulverized coal. Results from simultaneous measurements of the transmission of visible and near infra-red radiation by the pulverized coal are detailed next. Finally, the use of the experimental data in the "inverse radiation analysis" is discussed.

\subsection{Calibration of Detectors}

In order to interpret the voltage readings measured and recorded from the $\mathrm{CO}_{2}$-laser nephelometer experiments, it is first necessary to calibrate the detectors of the system. This involves the determination of the conversion factor whereby the output of the system (in $m V$ ) can be expressed in terms of the intensities of the radiation incident on the detector. For this purpose, spherical glass particles (Potter Industries Inc.) of diameters $81-135 \mu \mathrm{m}$ are used. Glass particles are chosen for the calibration for two reasons. They flow easily and can be used with little difficulty in a burette, thus removing the need for complicated flow devices of any sort. Secondly, the phase function of these particles determined from the Lorenz-Mie theory for a wavelength of $10.6 \mu \mathrm{m}$ yields a peak and trough within the first $15^{\circ}$. Thus, the experimental results obtained would enable not only the calibration of the detectors but also the exact angular position of the optical table when placed at a particular division of the scale. This can be done by comparing the angle of the peak and trough of the graph obtained from the experiment with that predicted by theory.

The experimental system used for the calibration has been described earlier (Bush, 1989; Bush et al., 1990). The apparatus consists of a $250 \mathrm{ml}$ burette which feeds the particles into the test-cell. This system produces a one dimensional planar flow of glass particles. Flow rate measurements are made using a stop watch. It is possible to vary the optical thickness of the flow by varying the flow of particles from the burette.

The optical thickness of the coal cloud is measured by recording the transmission of laser radiation. Then the larger aperture is mounted for scattering measurements and 
readings are taken from division 16 - corresponding to an angle of $6.1^{\circ}$ - to division 28 - corresponding to an angle of $10.6^{\circ}$ in steps of one division. The reason for limiting the measurements to the range represented by these two extremity angles is the increase in the fixed error made in measurements for larger angles.

Figure 7 depicts the angular distribution of the normalized power scattered by the spherical glass particles as obtained from Lorenz-Mie theory. The size distribution of the particles is obtained from scanning electron microscope photographs. Although diameters from 81 $\mu \mathrm{m}$ to $135 \mu \mathrm{m}$ were present in the sample, a good majority of the particles were in the size range of $97 \mu \mathrm{m}$ to $113 \mu \mathrm{m}$. The refractive index for these particles is obtained by linearly interpolating data available in the literature (Hsieh and $\mathrm{Su}, 1979$ ) for a wavelength of 10.6 $\mu \mathrm{m}$, and is expressed by the relation, $\tilde{n}_{10.6}=2.11-0.64 i$. The scattering phase function for the glass particles is first obtained for each narrow size range using the Lorenz-Mie theory and assuming a mean diameter for each range. The overall phase function is then calculated using the number density of the particles in each size range. This phase function is then used in the forward radiation analysis for planar media given earlier to obtain the angui ur power distribution depicted in Figure 7. Both first and second orders of the scattering of radiation are considered in this analysis.

From Figure 7, it can be seen that the normalized power drops to a minimum value at an angle of $6.8^{\circ}$ before rising to a secondary maximum at an angle of $8.6^{\circ}$. The angular distribution of the scattered intensity as recorded in the experiments is also plotted in this Figure and are expressed in terms of the output of the detector, $m V$. The angular distribution of scattered intensity obtained experimentally is of the same shape as the intensity values generated by the Lorenz-Mie theory. It is possible therefore, to match the theoretical and experimental data sets and thus scale the measurements obtained experimentally to express them in terms of intensities. Using this technique, a calibration factor of 0.25 is applied to scale the measurements obtained experimentally to the theoretical results. This factor accounts for the uncertainities arising from lens surface reflections and transmissions, detector window transmissions, laser beam irregularities etc.

\subsection{Coal Experiments}

\subsubsection{Scattering/Transmission Experiments at $10.6 \mu \mathrm{m}$}

Measurements with the pulverized coal using the $\mathrm{CO}_{2}$-laser nephelometer are made after the detectors have been calibrated. The experiments were carried out for the Blind Canyon coal for different size distributions. The proximate, ultimate and elemental analyses of the coal is listed in Table 1. The fluidized bed is filled to about two-thirds with the particles 
of the coal to be studied. The burette and glass test-cell are replaced by the coal test-cell. The optical table is rotated to position the detector roughily at about $0^{\circ}$. To obtain the exact zero position, the table is rotated in small steps till the detector output is maximum, as observed from a graphical display on the computer screen. First, the direct reading $\left(V_{0}\right)$ is recorded. Keeping the table stationary, the flow of particles is started until a steady flow rate is shown on the strip chart recorder. In this study flow rates of coal used were between 3 and $9 \mathrm{gm} / \mathrm{min}$. The detector reading is recorded in terms of the voltage $\left(V_{1}\right)$. A first estimate for the reduced optical thickness $(\tau)$ of the coal particle cloud is then given by the relation:

$$
i=\beta L\left(1-\frac{\omega \Phi(0) \Delta \Omega}{4 \pi}\right)=-\log _{e}\left(\frac{V_{1}}{V_{0}}\right)
$$

The angular distribution of the scattered intensity radiation is then recorded. For this, the smaller diameter aperture is replaced by the larger one and the measurements are made at the following angles $3.0^{\circ}, 3.8^{\circ}, 4.56^{\circ}, 5.3^{\circ}, 6.1^{\circ}, 7.98^{\circ}, 9.88^{\circ}, 11.78^{\circ}$ and $14.82^{\circ}$. This ensures that the angles at which the effect of scattering is the strongest are covered adequately. It is not possible to make measurements at angles smaller than $2.66^{\circ}$ as the finite size of the laser beam interferes with the detector set-up.

Figure 8 depicts the variation of optical thickness $(\tau)$, with flow-rate for the size distribution of $32-38 \mu \mathrm{m}$; similar results were obtained for other size distributions. From this figure, it can be seen that for low optical flow rates, the optical thickness of the particle cloud bears a linear relation to the mass flow rate, as expected. The linear dependence of the optical thickness on the flow rate is due to the increase in the number density of the particles in the cloud. With increasing flow, there are a greater number of particles to absorb and scatter the incident radiation. However, with further increase in particle number density, multiple scattering effects begin to appear. This is the reason for the deviation of the linear relationship between the optical thickness and the mass flow rate. Note that optical thickness is the product of the extinction coefficient and the physical length of the system, which is measured independently $(9.5 \mathrm{~mm})$.

\subsubsection{Inverse Radiation Analysis}

The data obtained from the scattering/transmission experiments should be processed to be able to recover the radiation properties of the coal particles such as the phase function $\left(\Phi_{\lambda}\right)$ and extinction $\left(\beta_{\lambda}\right)$ coefficient. This can be achieved by following an inverse radiation analysis. 
The experimental results for the radiation scattered by the pulverized coal particles are expressed in terms of the detector output voltages. There are then scaled and expressed in terms of intensities incident on the detector using the conversion factor obtained from the calibration of the system. The intensities so obtained depend on the solid angle and the illuminated area from which the radiation is incident on the detector. They are expressed in terms of the product of the scattering albedo $\omega$ and the phase function $\Phi(\theta)$ using the radiation model for cylindrical systems developed by Mengüç and Manickavasagam (1990).

The following is step-by-step description of the procedure followed to obtain the phase function from the experimental data:

- Reduced optical thickness for the cylindrical coal particle cloud based on the $\mathrm{CO}_{2}$-laser transmission is obtained from Equation (10).

- Based on preliminary studies, a rough estimate for the term $\left[1-\frac{\omega \Phi(\theta) \Delta \Omega}{4 \pi}\right]$ is found. An estimate of the value of the scattering albedo $\omega$ can be determined using the Lorenz-Mie theory. Table 3 gives the variation of scattering albedo with refractive index and diameter. The values for refractive index have been obtained from literature: $\tilde{n}=1.7-0.07 i$ from Brewster and Kunitomo (1984); $\tilde{n}=1.7-0.60 i$ (near infrared) and $\tilde{n}=2.5-1.2 i$ (far infrared) from Foster and Howarth (1968). Note that these values are different from each other, because of the different experimental techniques used to obtain them. It is clear from Table 2 that the value for $\omega$ is uniform for a wide range of particle sizes. For preliminary analysis, $\omega=0.5$ is chosen. The peak in the forward direction, $\Phi(0)$ is taken to be about 2000 and $\Delta \Omega=5 \times 10^{-4}$ to find $\left[1-\frac{\omega \Phi(\theta) \Delta \Omega}{4 \pi}\right]$ $\sim 0$ 36. Using this value with the measured value of the diameter of the cloud $L, a$ corrected optical thickness for the coal particle cloud is determined.

- The voltage readings obtained from scattering experiments are scaled to the normalized power (angular radiosity) distribution using the conversion factor determined from the calibration of the system. These readings are given in Table 3.

- Using the first-order scattering analysis for cylindrical media, the angular radiosity data are modified to eliminate the effects of geometry and the finite solid angle of the detectors. The result of this reduction, expressed in the form of the product $\omega \cdot \Phi(\theta)$ is comparable to the scattering phase function of the cloud of particles.

- Assuming $\omega=0.52$, the experimental phase function is estimated. Using the step-phase function approximation (Mengüç and Subramaniam, 1990), the Legendre coefficients of the phase function is determined. 
- Using this phase function, the effect of second order scatter:.ag on the angular radiosity distribution is then determined.

- This second-order contribution is subtracted from the experimentally determined radiosity. This gives the radiosity $\left(R_{1}(\theta)\right)$ based on the first-order scattering.

- This value of radiosity $R_{1}(\theta)$, is then reduced to the product $\omega \cdot \Phi(\theta)$ by eliminating the geometry and finite solid angle effects from the radiation analysis given in Section 2. The results for the Blind Cariyon coal of various size ranges are shown in Tables 4 through 9.

- Assuming $\omega=0.52$, a new phase function is determined. The first three coefticients of the new Legendre polynomial expansion are given in Table 10.

It is possible to conclude from these results that as the Legendre coefficients of the full phase function expansion remain constant with change in diameter, the same expression for phase function can be used for different size distributions. Also, the peak values of the phase function together with the Legendre coefficients suggest that the particles are highly forward scattering. The second order scattering analysis yields phase function values that are $0.25 \%$ of that for the first order. Therefore, it can be concluded that first order scattering is sufficient to analyze the data obtained from experiments.

In the inverse analysis presented, it was not possible to separate the albedo from the phase function; therefore, a constant uniform value is used. This is an arbitrary choice, and needs more careful consideration.

\subsubsection{Transmission Measurements at Visible Wavelengths}

Transmission of monochromatic light through the coal particle cloud was measured after the completion of the scattering/transmission measurements with the $\mathrm{CO}_{2}$-laser nephelometer. The arc lamp is sparked a while before the measurements are made to enable it to reach its peak power. The wavelength dial of the monochromator is set to the desired number. The incident intensity for each desired wavelength is then recorded. Measurements are made at the wavelengths of $438.7 \mathrm{~nm}, 548.6 \mathrm{~nm}, 579.6 \mathrm{~nm}$ and $700 \mathrm{~nm}$. These wavelengths represent the peaks in the output spectrum of the Mercury Arc lamp except for $700 \mathrm{~nm}$, which is used as it is the highest visible wavelength Note that these values of the wavelength refer to the setting on the monochromator dial and they may be a difference of $3 \mathrm{~nm}$ with the exact wavelengths of the Mercury lamp. A lock-in amplifier is used to eliminate the effect of radiation from the surroundings and provides a digital readout of the intensity in $m V$. 
The coal flow is then started and the transmitted intensities at the various wavelengths recorded. Voltage readings obtained with and without coal flow are used to determine a reduced optical thickness from Equation (10). It is worthwhile to note that because the wavelengths being considered are small, the size parameter for the coal particles used is large; therefore, the scattered intensity distribution has a high forward peak. In addition to this, the collection angle of the optic fibre used to carry the transmitted light is about $12^{\circ}$. Be.ause of this all the light scattered in the forward direction is measured, meaning that no energy is lost because of scattering (in-scattering gain is equal $t$ out-scattering loss). Under this situation, it is possible to say that any portion of the energy that is attenuated is because of the absorption of radiation by the coal particles.

The optical thicknesses obtained from Equation (10) are normalized to account for the difference in the flow rates for each size range and are given in Table 12. From these results, it is observed that for a particular size distribution, the optical thickness starting from the lower end of the spectrum $(438.7 \mathrm{~nm})$, drops to a minimum and subsequently increases again at $700 \mathrm{~nm}$. The exception is the range $0.25 \mu \mathrm{m}$ which is probably due to the agglomeration of particles. This trend is observed for all the size distributions. The minimum optical thickness is obtained at the wavelengths of $438.7 \mathrm{~nm}$ and $548.6 \mathrm{~nm}$.

It can also be seen that as the size of the particles increase, the optical thickness first decreases for a given wavelength, and then increases slightly for the 53-63 $\mu \mathrm{m}$ particles. For the wavelength of $579.6 \mathrm{~nm}$, the optical thickness of the cloud containing the particles of the size range $00-25 \mu \mathrm{m}$ is 0.181 (mean size parameter ${ }^{1}$ is 54 ), while for the ranges $25-32 \mu \mathrm{m}$ (mean size parameter of 152), 32-38 $\mu \mathrm{m}$ (190), 38-45 $\mu \mathrm{m}$ (222), 45-53 $\mu \mathrm{m}$ (266) and 53-64 $\mu \mathrm{m}(314)$ it is $0.134,0.123,0.097,0.056$ and 0.069 respectively. It is possible to explain the decrease in optical thickness by noting that the number density of the particles in the cloud is large for smaller mean diameters, which yields a higher absorption coefficient and medium optical thickness.

Also listed in Table 12 are the size parameters at the given wavelength for the mean diameter of each size distribution. The mean diameters used are $D_{10}$, with values of $10,28,35$, 41,49 and $57 \mu \mathrm{m}$ with increasing size range. The size paramters for the particles considered vary widely from 45 to 415 . The measurements show that even for size parameters of the same order, the optical thicknesses vary. For a size parameter of 222 ( D: 38-45 $\mu \mathrm{m}$ and $\lambda=579.6 \mathrm{~nm}$ ) the optical thickness is 0.0969 while for a size parameter of 220 (D: $45-53 \mu \mathrm{m}$ and $\lambda=700 \mathrm{~nm}$ ) the optical thickness is 0.0846 . Another example can be seen for a size

\footnotetext{
${ }^{1}$ Note that size parameter, by definition, is for homogeneous spherical particles; the reason for discussing size parameters here is only for comparison purposes
} 
parameter of 160 (D:25-32 $\mu \mathrm{m}$ and $\lambda=548.6 \mathrm{~nm}$ ) for which the optical thickness is 0.1100 while for 157 ( $D: 32-38 \mu \mathrm{m}$ and $\lambda=700 \mathrm{~nm}$ ) it is 0.1590 .

From these measurements it is possible to conclude that absorption is not only a function of the size parameter but is also wavelength dependent in the visible range.

\section{CONCLUSIONS AND RECOMMENDATIONS}

From the experiments conducted and the "inverse radiation analysis" performed on the measured values, it is possible to draw the following conclusions:

- The inverse radiation analyses performed for the results obtained from the $\mathrm{CO}_{2}$ laser experiments yielded the same values for the Legendre coefficients of the phase function expansion for the various size ranges. This suggests that the same highly-forward phase function can be used for all the different diameters used in this study. The phase function has a peak of about 1100 in the forward direction.

- Variation of optical thickness with low flow rates is linear if the number density of the particles is small. However, for higher flow rates this relation becomes an inverse exponential function because of the increased contribution of forward scattering by the particles. As the particles are highly forward scattering, the contribution of second order scattering can be neglected for optical thicknesses less than 1.

- The absorption of visible radiation displayed a wavelength dependent behaviour. Optical thickness measurements carried out with the monochromator indicate that for increasing values of wavelength, coal absorption coefficient decreases slightly at green and then increases again. This trend is observed for five out of the six size distributions considered.

However, it is not possible to claim that these observations will be the same for different coal rank and for char particles. Further studies are required to address these points. Suggestions for future work in determining the radiative properties of pulverized-coal particles are listed below:

- Experimental techniques can be improved to obtain more detailed information for the forward peak of the phase function.

- The experiments can be extended to include other wavelengths. 
- Particles of smaller diameter should be considered as the experiments conducted with smaller size particles are more sensitive to wavelength and particle optical properties and shape. However, care should be taken to avoid any agglomeration of particles.

- The effect of pyrolysis and combustion characteristics on the properties should also be studied. 


\section{REFERENCES}

Agarwal, B. and Mengüç, M.P. "Forward and Inverse Analysis of Single and Multiple Scattering of Collimated Radiation in an Axisymmetric System", International Journal of Heat and Mass Transfer, 1990 (in press).

Altenkirch, R.A., Peck, R.E. and Chen, S.L. "Fluidized-bed Feeding of Pulverized-coal", Powder Tcchnology, Vol. 20, pp. 189-196, 1978.

Barber, P. and Yeh, C., "Scattering of Electromagnetic Waves by Arbitrarily Shaped Dielectric Bodies", Applied Optics, Vol. 14, pp.2864-2872, 1975.

Blokh, A. G. Heat Transfer in Steam Boiler Furnaces, Hemisphere Publishing Corp., Washington, D.C. 1988. (Russian edition 1984.)

Blokh, A. G. and Burak, L.D. "Radiation Characteristics of Solid Fuels", Teploenengerika, Vol. 20 (8), pp. 48-52, 1973.

Bohren, C.F. and Huffman, D.R. Absorption and Scattering of Light by Small Particles, Wiley, New York, 1983.

Brewster, M.Q. and Kunitomo, T. "The Optical Constants of Coal, Char, and Limestone", Journal of Heat Transfer, Vol. 106, pp. 678-683, 1984.

Bush, M. " $\mathrm{A} \mathrm{CO}_{2}$ Laser Nephelometer to Determine The Radiative Properties of Pulverized Coal Particles", MSME Thesis, Department of Mechanical Engineering, University of Kentucky, Lexington, Kentucky, 1989.

Bush, M., d'Sa, D.A., Manickavasagam, S., Mengüç, M.P. "Design and Calibration of a $\mathrm{CO}_{2}$-Laser Nephelometer to Measure the Radiative Properties of Coal Iarticles", Spring 1990 Technical Meeting, Central States Section, Combustion Institute, Cincinnati, OH, pp. 259-264, 1990.

Ergun, S., McCartney, J.T. and Walline, R.E. "Absorption of Ultra-violet and Visible Light by Ultra-thin Sections of Coal", Fuel, XL(2), pp. 109-117, 1961.

Field, M.A., Gill, D.W., Morgan, B.B. and Hawksley, P.G.W. Combustion of Pulverized Coal, The British Coal Utilization Research Association, Leatherhead, Surrey, England, 1967. 
Flagan, R.C. "Sub-micron Particles from Coal Combustion", Seventeenth Symposium (International) on Combustion, 97-104, The Combustion Institute, Pittsburgh, PA, 1979.

Foster, C.J. and Howarth, C.R. "Optical Constants of Carbons and Coals in the Infrared", Carbon, Vol. 6, pp. 719-729, 1968.

Grosshandler, W.L. and Monterio, S.L.P. "On the Spectral Emissivity of Pulverized Coal and Char", Journal of Heat Transfer, Vol. 104, pp. 587-593, 1981.

Hsieh, C.K. and Su, K.C. "Thermal Radiative Properties of Glass from 0.32 to $206 \mu \mathrm{m}$ ", Journal of Solar Energy, Vol. 22, pp. 37-43, 1979.

IIuntjens, F.J. and van Krevelen, D.W. "Chemical Structures and Properties of Coal: IIReflectance", Fuel, Vol. 33, pp. 88-103, 1954.

Kerker, M. The Scattering of Light, Academic Press, New York, 1969.

McCartney, J.T. and Ergun, S. "Optical Properties of Graphite and Coal", Fuel, Vol. 37, pp. 272-282, 1958.

Mengüç, M.P. and Subramaniam, S. "A Step-Phase Function Approximation for the Experimental Determination of the Effective Scattering Phase Function of Particles", Journal of Quantitative Spectroscopy and Radiative Transfer, Vol. 43, pp. 253-265, 1989.

Mengüç, M.P. and Manickavasagam, S., (1990). "Analytical Solution of Inverse Radiation Problems in Nonhomogeneous Media", in preparation.

Mugnai, A. and Wiscombe, W.J. "Scattering for Nonspherical Chebyshev Particles: Variability in Angular Scattering Patterns", Applied Optics, Vol. 28, 3061-3073, 1989.

Özişik, M.N. Radiative Transfer and Interactions With Conduction and Convection, Wiley, New York, 1973.

Purcell, E.M. and Pennypacker, C.R. "Scattering and Absorption by Non-Spherical Dielectric Grains", Astrophysics Journal, Vol. 186, pp. 705-714, 1973.

Sarofim, A.F. and Hottel, H.C. "Radiative Transfer in Combustion Chambers: Influence of Alternative Fuels", Proceedings of Sixth International Heat Transfer Conference, Toronto, Canada, Vol. 6, pp. 199-217, 1978. 
Smoot, L.D. "Modeling of Coal Combustion Processes", Progress in Energy and Combustion, Vol. 10, pp. 229-272, 1984.

Smoot, L.D. and Smith, P.J. Coal Combustion and Gasificalion, Plenum Press, New York, 1985.

Solomon, P.R. Carangclo, R.M., Best, P.E., Markham, J.R. and Hamblen, D.G. "The Spectral Emmittance of Pulverized-Coal and Char ', Twenty-first Symposium (International) on Conibustion, The Combustion Institute, Pittsburgh, pp.437-446, 1986.

Solomon, P.R., Cacangelo, R.M., Best, P.E., Markham, J.R. and Hamblen, D.G. "Analysis of Particie Emittance, Composition, Size and Temperature by FT-IR Emission/Transmission Spectroscopy", Fuel, Vol. 66, pp. 897-908, 1987.

Stull, V.R. and Plass. G.N. "Emissivity of Dispersed Carbon Particles" Journal of the Optical Society of Amerisa, 50(2):65- $\imath 1,1960$.

van de Hulst, H.C. Light Scattering by Small Particles, Dover, New York, 1981, 1957.

Viskanta, R. and Mengüç, M.P. "Radiation Heat Transfer in Combustion Systems", Progress in Enemy and Combustion Sciences, Vol. 13, pp. 97-160, 1987.

Waterman, F.C. "Matrix Formulation of Electromagnetic Scattering", Proceedings of IEEE, $53,805,1965$.

Wiscombe, W.J. "Improved Mie Scattering Algorithms", Applied Optics, 19, pp. 1505-1509, 1980.

Wiscombe, W.J. and Mugnai, A. Single Scattering From Nonspherical Chebyshev Particles: A Compendium of Calculations, NASA Reference Publication \# 1157, Washingion D.C., 1586.

Yeh, C. and Mei, K. "On The Scattering From Ar'bitrarily Shaped Inhomogeneous Particles - Exaci Solution", iight Scattering from Irregularly Shaped Particles, D.W. Schuerman (Ed), pp. 201.206, Plenum Press, New York, 1980. 
Table 1: Proximate, Ultimate and Elemental Analysis of Blind Canyon Coal

$\begin{array}{lll}\text { Seam Name } & : & \text { BLIND CANYON } \\ \text { PSU Number } & : & \text { PSOC-1503 } \\ \text { Apparent Rank } & : & \text { HVB }\end{array}$

\begin{tabular}{|l|r|r|}
\hline \multicolumn{3}{|c|}{ PROXIMATE ANALYSIS } \\
\hline & As Received & Dry \\
\hline \hline \% Moisture & 10.35 & $*$ \\
\% Ash & 3.51 & 3.91 \\
\% Volatile & 41.89 & 46.73 \\
\% Fixed Carbon & 44.25 & 49.36 \\
\hline
\end{tabular}

\begin{tabular}{|l|r|r|}
\hline \multicolumn{3}{|c|}{ ULTIMATE ANALYSIS } \\
\hline & As Received & Dry \\
\hline \% Ash & 3.51 & 3.91 \\
\% Carbon & 69.27 & 77.27 \\
\% Hydrogen & $5.29 \dagger$ & 5.90 \\
\% Nitrogen & 1.33 & 1.48 \\
\% Total Sulphur & 0.48 & 0.54 \\
\% Oxygen (differeace) & $9.77^{\dagger}$ & 10.90 \\
\hline
\end{tabular}

$\dagger$ Excludes Moisture

\begin{tabular}{|l|r|}
\hline \multicolumn{2}{|c|}{ ELEMENTAL ANALYSIS } \\
\hline & Dry \\
\hline \hline \% Carbon & 77.2 \\
\% Hydrogen & 5.85 \\
\% Nitrogen & 1.48 \\
\% Organic Sulphur & 0.52 \\
\% Oxygen (difference) & 10.49 \\
\% Mineral Matter & \\
(incl. 0.04\% FeS 2 ) & 4.46 \\
\hline
\end{tabular}


Table 2: Variation of tlbede IVith Diameter for Ceal

Coal Type : BLIND CANYON

\begin{tabular}{|c|c|c|c|}
\hline Diameter & \multicolumn{3}{|c|}{ Refractive Index } \\
\hline $\mathrm{d}$ & $\mathrm{n}=1.7 \cdot 0.0 \mathrm{i} i$ & $\mathrm{n}=1.7 \cdot 0.60 \mathrm{i}$ & $\mathrm{n}=2.5 \cdot 1.20 i$ \\
\hline \hline 10 & 0.7786 & 0.4607 & 0.5245 \\
28 & 0.5705 & 0.5139 & 0.5843 \\
35 & 0.5181 & 0.5254 & 0.5937 \\
41 & 0.5412 & 0.5327 & 0.5996 \\
49 & 0.5176 & 0.5400 & 0.6057 \\
58 & 0.5324 & 0.5463 & 0.6109 \\
\hline
\end{tabular}

Table 3: Normalized Power Values For Coal

Coal Type : BLIND CANYON

\begin{tabular}{|c|c|c|c|c|c|c|}
\hline Angle & \multicolumn{7}{|c|}{ Size Distribution } \\
\hline degrees & Diameter & Diameter & Diameter & Diameter & Diameter & Diameter \\
\hline & $00-25$ & $25-32$ & 32.38 & $38-45$ & $45-53$ & $53-64$ \\
\hline \hline 3.06 & 0.370 & 0.144 & 0.359 & 0.397 & 0.429 & 0.437 \\
3.82 & 0.288 & 0.131 & 0.285 & 0.335 & 0.364 & 0.340 \\
4.58 & 0.230 & 0.115 & 0.228 & 0.276 & 0.285 & 0.258 \\
5.35 & 0.197 & 0.103 & 0.191 & 0.223 & 0.246 & 0.209 \\
6.11 & 0.177 & 0.086 & 0.155 & 0.189 & 0.194 & 0.171 \\
8.02 & 0.124 & 0.058 & 0.101 & 0.113 & 0.115 & 0.100 \\
9.93 & 0.082 & 0.041 & 0.064 & 0.071 & 0.071 & 0.060 \\
11.84 & 0.054 & 0.031 & 0.043 & 0.046 & 0.046 & 0.040 \\
14.90 & 0.035 & 0.019 & 0.025 & 0.028 & 0.027 & 0.025 \\
\hline
\end{tabular}


Table 4: Albedo $(\omega) \times$ Phase Function $(\Phi(\theta))$ Values For Coal

$\begin{array}{lll}\text { Coal Type } & : & \text { BLIND CANYON } \\ \text { Size Distribution } & : & 0-25 \mu \mathrm{m} \\ \text { Optical Thickness } & : & 0.71 \\ \text { Reflected Reading } & : & 2.132 \mathrm{mV} \\ \text { Time Constant } & : & 3 \text { seconds }\end{array}$

\begin{tabular}{|c|c|c|c|c|c|}
\hline Angle & Number of & \multicolumn{3}{|c|}{ Experimental Readings } & $\omega \times \Phi(\theta)$ \\
\hline degrees & & $\mathrm{ml}^{\prime}$ & Sensitivity (mV) & Standard Deviation & \\
\hline 3.06 & 8 & 3.0420 & 10 & 0.10 & 12.12 \\
\hline 3.82 & 10 & 2.3728 & 25 & 0.08 & 9.45 \\
\hline 4.58 & 12 & 1.8917 & 25 & 0.03 & 7.53 \\
\hline 5.35 & 14 & 1.6195 & 25 & 0.03 & 6.45 \\
\hline 6.11 & 16 & 1.4585 & 25 & 0.03 & 5.81 \\
\hline 8.02 & 21 & 1.0196 & 10 & 0.02 & 4.06 \\
\hline 9.93 & 26 & 0.6750 & 10 & 0.01 & 2.69 \\
\hline 11.84 & 31 & 0.4430 & 10 & 0.01 & 1.76 \\
\hline 14.90 & 39 & 0.2876 & 10 & 0.01 & 1.14 \\
\hline
\end{tabular}

Table 5: Albedo $(\omega) \times$ Phase Function $(\Phi(\theta))$ Values for Coal

$\begin{array}{lll}\text { Coal Type } & : & \text { BLIND CANYON } \\ \text { Size Distribution } & : 25.32 \mu \mathrm{m} \\ \text { Optical Thickness } & : 0.3653 \\ \text { Reflected Reading } & : 1.775 \mathrm{mV} \\ \text { Time Constant } & : \quad 3 \text { seconds }\end{array}$

\begin{tabular}{|c|c|c|c|c|c|}
\hline Angle & Number of & \multicolumn{3}{|c|}{ Experimental Readings } & $\omega \times \Phi(\theta)$ \\
\hline degrees & & $\mathrm{mV}$ & Sensitivity (mV) & Standard Deviation & \\
\hline 3.06 & 8 & 0.9870 & 25 & 0.04 & $6.6 i$ \\
\hline 3.82 & 10 & 0.9005 & 25 & 0.06 & 6.08 \\
\hline 4.58 & 12 & 0.7910 & 25 & 0.02 & 5.34 \\
\hline 5.35 & 14 & 0.7040 & 25 & 0.03 & 4.75 \\
\hline 6.11 & 16 & 0.5896 & 10 & 0.02 & 3.98 \\
\hline 8.02 & 21 & 0.4000 & 10 & 0.01 & 2.70 \\
\hline 9.93 & 26 & 0.2830 & 10 & 0.01 & 1.91 \\
\hline 11.84 & 31 & 0.2002 & 10 & 0.00 & 1.42 \\
\hline 14.90 & 39 & 0.1287 & 2.5 & 0.00 & 0.87 \\
\hline
\end{tabular}


Table 6: Albedo $(\omega) \times$ Phase Function $(\Phi(\theta))$ Values for Coal

$\begin{array}{lll}\text { Coal Type } & : & \text { BLIND CANYON } \\ \text { Size Distribution } & : & 32-38 \mu \mathrm{m} \\ \text { Optical Thickness } & : & 0.3540 \\ \text { Reflected Reading } & : & 1.775 \mathrm{mV} \\ \text { Time Constant } & : & 3 \text { seconds }\end{array}$

\begin{tabular}{|c|c|c|c|c|c|}
\hline Angle & Number of & \multicolumn{3}{|c|}{ Experimental Readings } & $\omega \times \Phi(\theta)$ \\
\hline degrees & & $\mathrm{mV}$ & Sensitivity (mV) & Standard Deviation & \\
\hline 3.06 & 8 & 2.4588 & 25 & 0.07 & 16.96 \\
\hline 3.82 & 10 & 2.3728 & 25 & 0.06 & 13.46 \\
\hline 4.58 & 12 & 1.8917 & 25 & 0.04 & 10.76 \\
\hline 5.35 & 14 & 1.6195 & 25 & 0.02 & 9.02 \\
\hline 6.11 & 16 & 1.4585 & 25 & 0.02 & 7.34 \\
\hline 8.02 & 21 & 1.0196 & 10 & 0.01 & 4.75 \\
\hline 9.93 & 26 & 0.6750 & 10 & 0.01 & 3.02 \\
\hline 11.84 & 31 & 0.4430 & 10 & 0.01 & 2.01 \\
\hline 14.90 & 39 & 0.2876 & 2.5 & 0.00 & 1.17 \\
\hline
\end{tabular}

Table i: Albedo $(\omega) \times$ Phase Function $(\Phi(\theta))$ Values for Coal

$\begin{array}{lll}\text { Coal Type } & : & \text { BLIND CANYON } \\ \text { Size Distribution } & : & 38.45 \mu \mathrm{m} \\ \text { Optical Thickness } & : & 0.4475 \\ \text { Reflected Reading } & : & 1.775 \mathrm{mV} \\ \text { Time Constant } & : & 3 \text { seconds }\end{array}$

\begin{tabular}{|c|c|c|c|c|c|}
\hline Angle & Number of & \multicolumn{3}{|c|}{ Experimental Readings } & $\omega \times \Phi(\theta)$ \\
\hline degrees & & $\mathrm{mV}$ & Sensitivity (mV) & Standard Deviation & \\
\hline 3.06 & 8 & 2.7172 & 25 & 0.06 & 16.17 \\
\hline 3.82 & 10 & 2.2980 & 25 & 0.07 & 13.67 \\
\hline 4.58 & 12 & 1.8905 & 25 & 0.03 & 11.25 \\
\hline 5.35 & 14 & 1.5300 & 25 & 0.03 & 9.10 \\
\hline 6.11 & 16 & 1.2925 & 25 & 0.03 & 7.69 \\
\hline 8.02 & 21 & 0.7725 & 25 & 0.01 & 4.59 \\
\hline 9.93 & 26 & 0.4862 & 10 & 0.01 & 2.89 \\
\hline 11.84 & 31 & 0.3128 & 10 & 0.01 & 1.86 \\
\hline 14.90 & 39 & 0.1910 & 10 & 0.00 & 1.13 \\
\hline
\end{tabular}


Table 8: Albedo $(\omega) \times$ Phase Function $(\Phi(\theta))$ Values for Coal

$\begin{array}{lll}\text { Coal Type } & : & \text { BLIND CANYON } \\ \text { Size Distribution } & : 45.53 \mu \mathrm{m} \\ \text { Optical Thickness } & : & 0.5626 \\ \text { Reflected Reading } & : & 1.775 \mathrm{mV} \\ \text { Time Constant } & : & 3 \text { seconds }\end{array}$

\begin{tabular}{|c|c|c|c|c|c|}
\hline Angle & Number of & \multicolumn{3}{|c|}{ Experimental Readings } & $\omega \times \Phi(\theta)$ \\
\hline degrees & & $\mathrm{mV}$ & Sensitivity (mV) & Standard Deviation & \\
\hline 3.06 & 8 & 2.9398 & 25 & 0.04 & 15.48 \\
\hline 3.82 & 10 & 2.4950 & 25 & 0.04 & 13.14 \\
\hline 4.58 & 12 & 1.9545 & 25 & 0.04 & 10.29 \\
\hline 5.35 & 14 & 1.6865 & 25 & 0.03 & 8.88 \\
\hline 6.11 & 16 & 1.3290 & 25 & 0.03 & 6.99 \\
\hline 8.02 & 21 & 0.7865 & 25 & 0.01 & 4.14 \\
\hline 9.93 & 26 & 0.4875 & 25 & 0.01 & 2.57 \\
\hline 11.84 & 31 & 0.3160 & 10 & 0.00 & 1.66 \\
\hline 14.90 & 39 & 0.1820 & 10 & 0.00 & 0.96 \\
\hline
\end{tabular}

Table 9: Albedo $(\omega) \times$ Phase Function $(\Phi(\theta))$ Values

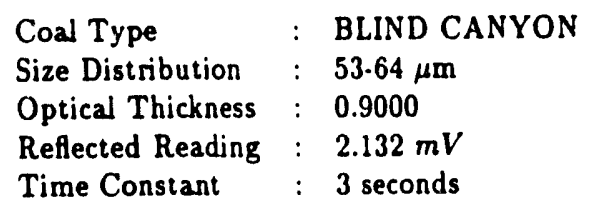

\begin{tabular}{|c|c|c|c|c|c|}
\hline Angle & Number of & \multicolumn{3}{|c|}{ Experimental Readings } & $\omega \times \Phi(\theta)$ \\
\hline degrees & & $\mathrm{mV}$ & Sensitivity $(\mathrm{mV})$ & Standard Deviation & \\
\hline 3.06 & 8 & 3.5970 & 25 & 0.05 & 13.48 \\
\hline 3.82 & 10 & 2.7950 & 25 & 0.04 & 10.47 \\
\hline 4.58 & 12 & 2.1255 & 25 & 0.03 & 7.96 \\
\hline 5.35 & 14 & 1.7180 & 25 & 0.02 & 6.44 \\
\hline 6.11 & 16 & 1.4065 & 25 & 0.02 & 5.27 \\
\hline 8.02 & 21 & 0.8206 & 10 & 0.01 & 3.07 \\
\hline 9.93 & 26 & 0.4946 & 10 & 0.01 & 1.85 \\
\hline 11.84 & 31 & 0.3284 & 10 & 0.01 & 1.23 \\
\hline 14.90 & 39 & 0.2038 & 10 & 0.00 & 0.76 \\
\hline
\end{tabular}


Table 10: Inverted Legendre Polynomial Coefficients for Albedo $\times$ Phase Function

Coal Type : BLIND CANYON

\begin{tabular}{|c|c|c|c|c|}
\hline Size Distribution & $\mathrm{a}_{1}$ & $\mathrm{a}_{2}$ & $\mathrm{a}_{3}$ & $\Phi(0)$ \\
\hline \hline $00-25$ & 2.995 & 4.983 & 6.974 & 1111 \\
$25-32$ & 2.993 & 4.972 & 6.936 & 1072 \\
32.38 & 2.996 & 4.989 & 7.014 & 1085 \\
$38-45$ & 2.993 & 4.970 & 6.933 & 1058 \\
$45-53$ & 2.994 & 4.975 & 6.941 & 1110 \\
$53-64$ & 2.997 & 4.994 & 7.032 & 1112 \\
\hline
\end{tabular}

Table 11: Variation of Optical Thickness IVith Wavelength

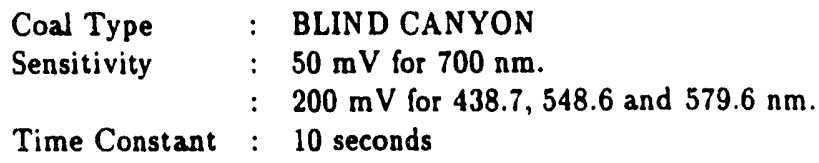

\begin{tabular}{|c|c|c|c|c|c|c|c|}
\hline \multirow{2}{*}{ Wavelength } & \multicolumn{5}{|c|}{ Optical Thickness for the Size Distributions } \\
\cline { 3 - 8 } & Diam. & Diam. & Diam. & Diam. & Diam. & Diam. \\
\hline $\mathrm{nm}$ & Color & 00.25 & $25-32$ & $32-38$ & 38.45 & $45-53$ & 53.64 \\
\hline \hline 438.7 & Blue & 0.124 & 0.099 & 0.102 & 0.066 & 0.041 & 0.068 \\
548.6 & Green & 0.075 & 0.110 & 0.098 & 0.067 & 0.037 & 0.066 \\
579.6 & Yellow & 0.181 & 0.134 & 0.123 & 0.097 & 0.056 & 0.069 \\
700.0 & Red & 0.134 & 0.166 & 0.159 & 0.124 & 0.085 & 0.074 \\
\hline
\end{tabular}

\begin{tabular}{|c|c|c|c|c|c|c|c|}
\hline \multirow{2}{*}{ Wavelength } & \multicolumn{5}{|c|}{ Size Parameters for the Mean Diameters } \\
\cline { 3 - 8 } & Diam. & Diam. & Diam. & Diam. & Diam. & Diam. \\
\hline nm & Color & $00-25$ & $25-32$ & $32-38$ & $38-45$ & $45-53$ & $53-64$ \\
\hline \hline 438.7 & Blue & 72 & 201 & 251 & 294 & 351 & 415 \\
548.6 & Green & 57 & 160 & 200 & 235 & 281 & 332 \\
579.6 & Yellow & 54 & 152 & 190 & 222 & 266 & 314 \\
700.0 & Red & 45 & 126 & 157 & 184 & 220 & 260 \\
\hline
\end{tabular}




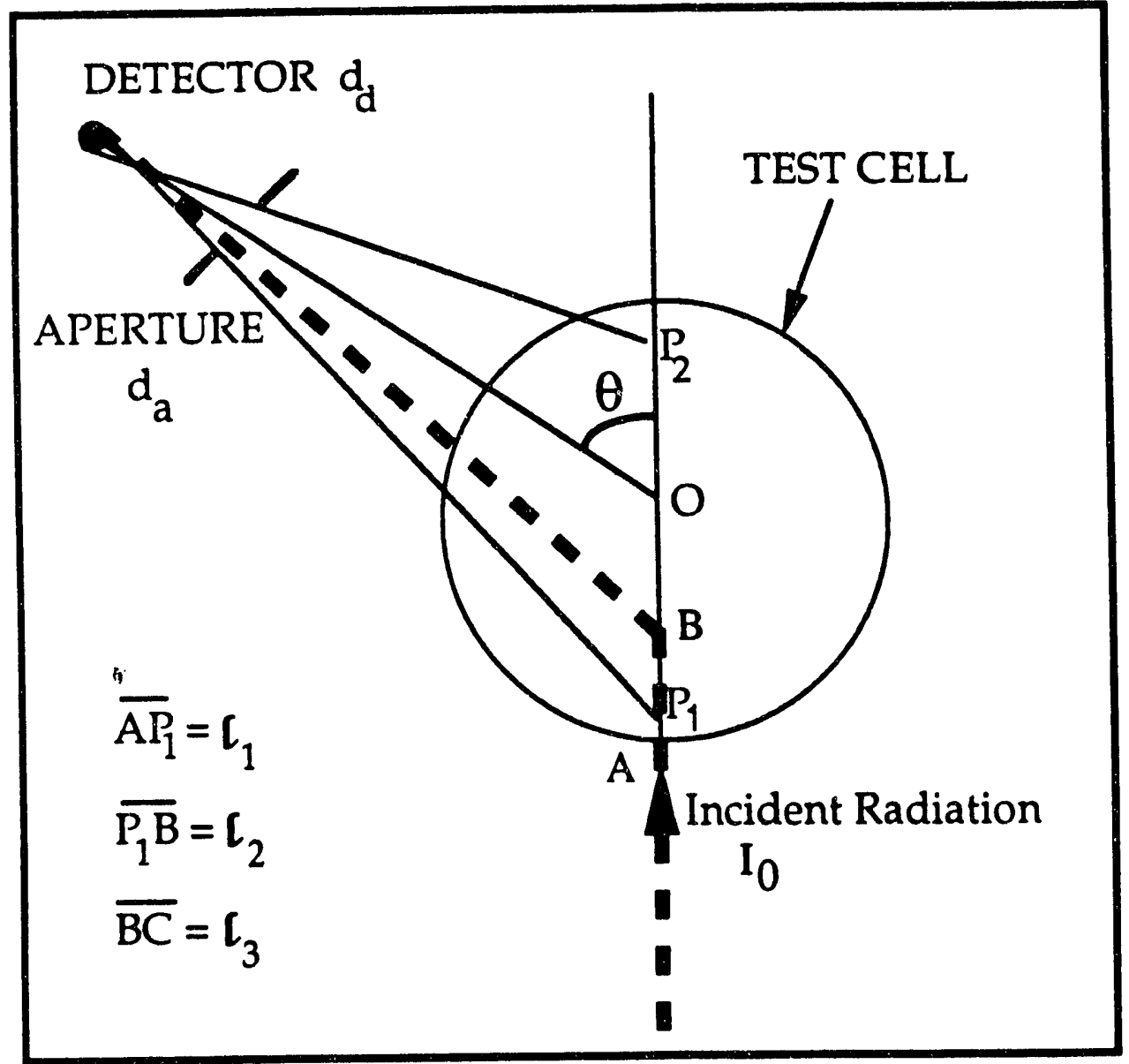

fig. 1.

First Order Scattering from a Cylindrical Particle Cloud 


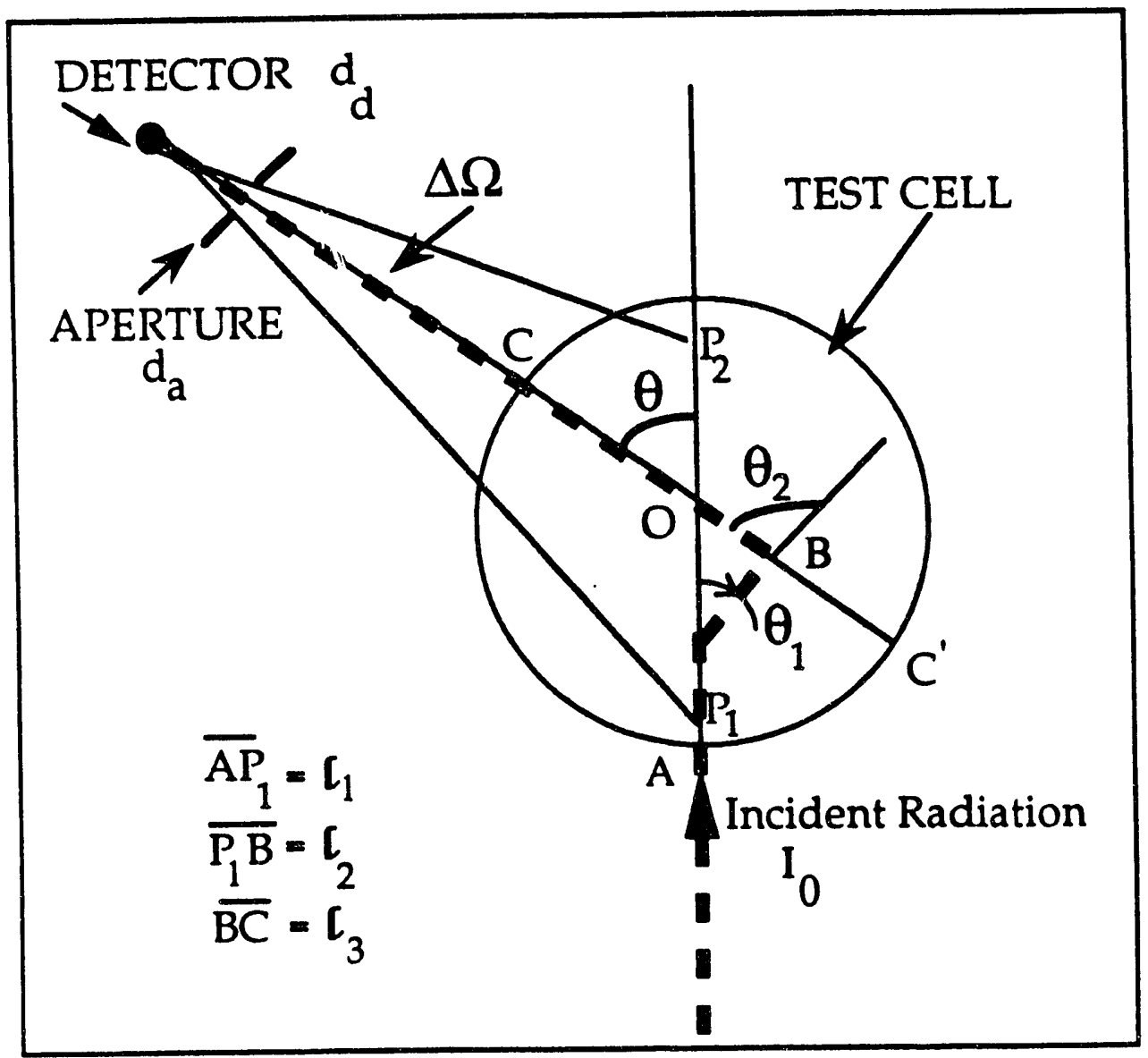

Figure 2 Second Order Scattering from a Cylindrical Particle Cloud 


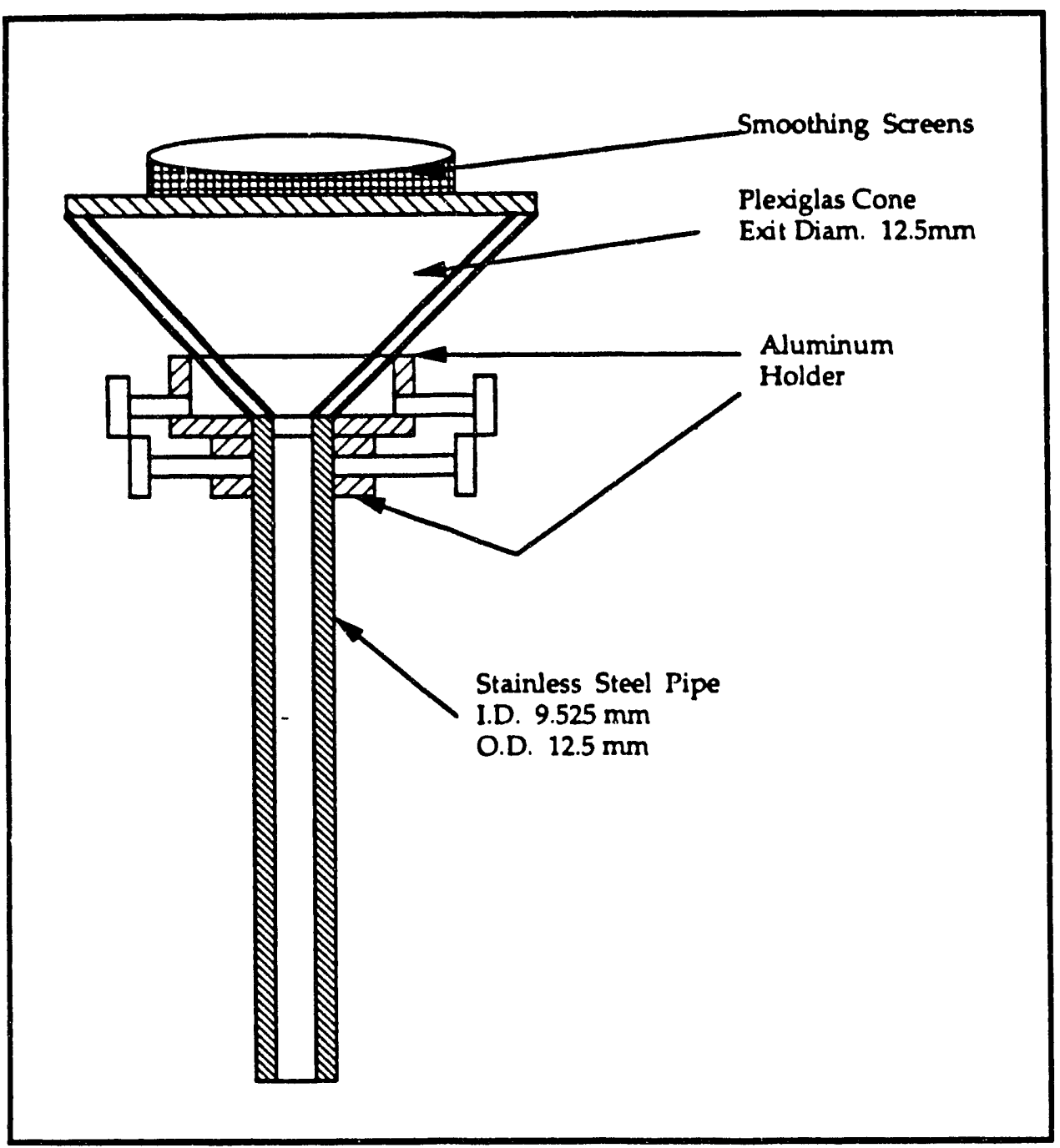

Figure 3 Schematic for the Cylindrical Coal Test-Cell 


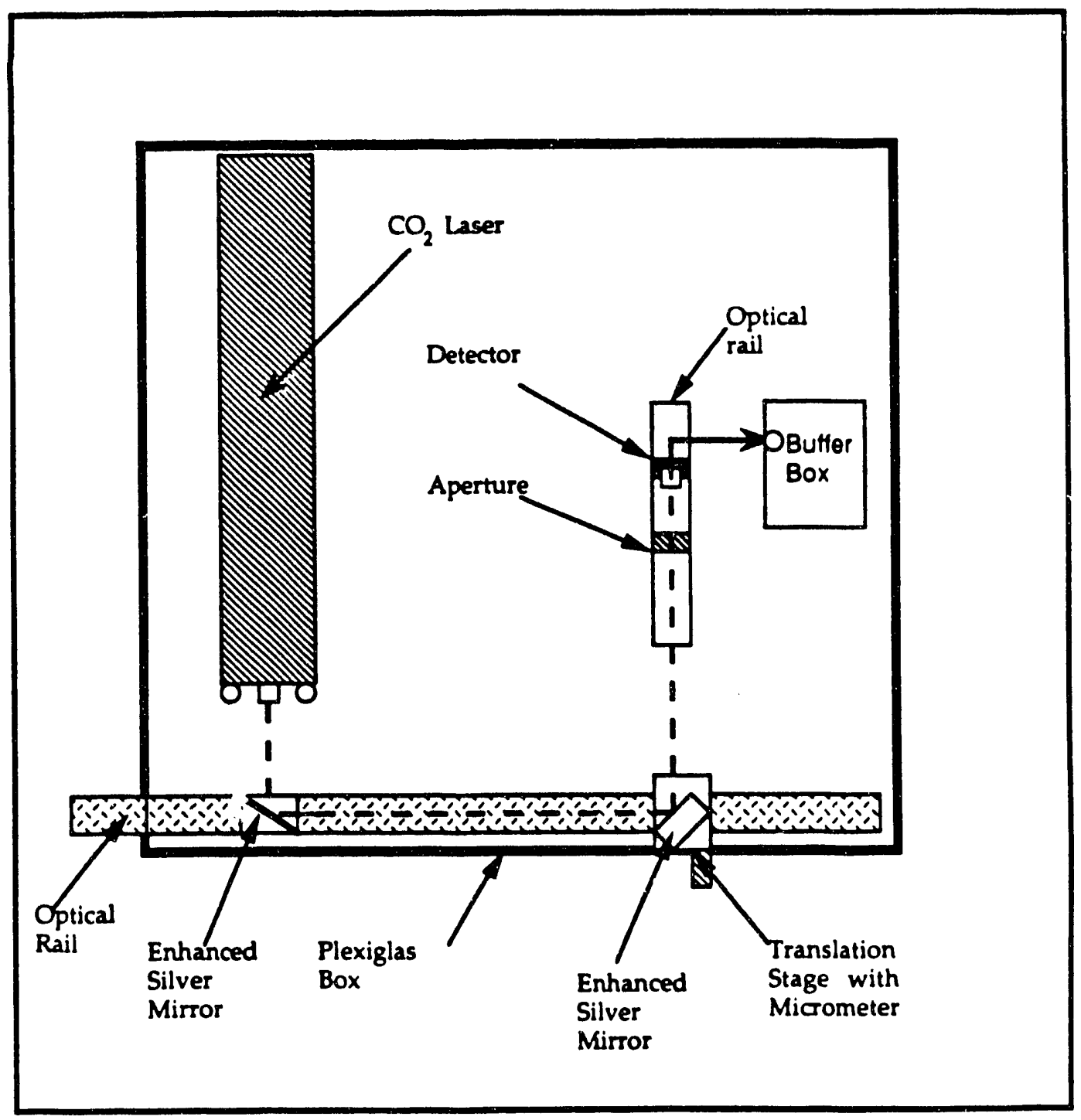

Figure 4 : Monitoring $\mathrm{CO}_{2}$-Laser Power 


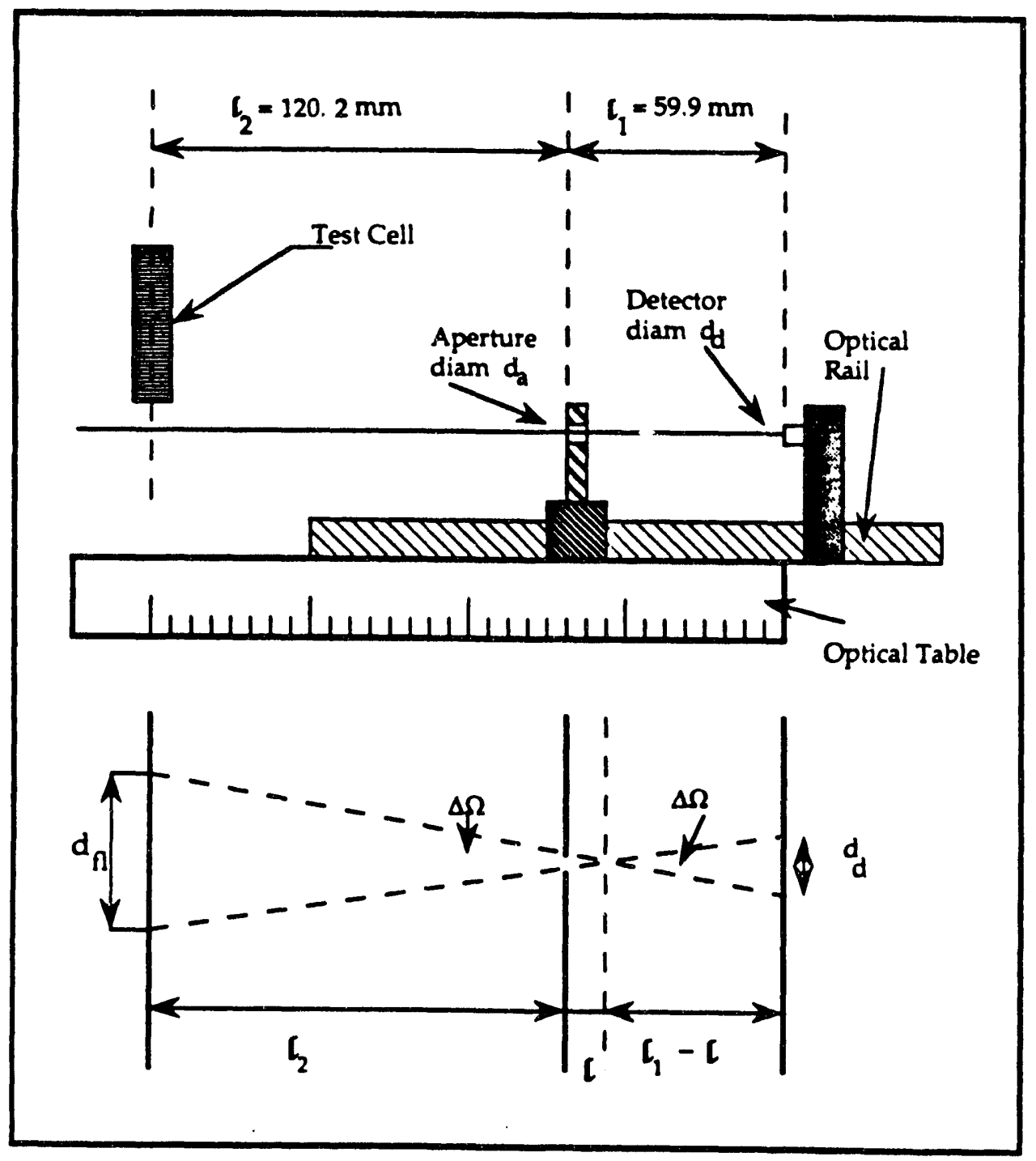

Figure 5 The Aperture-Detector Set-Up 


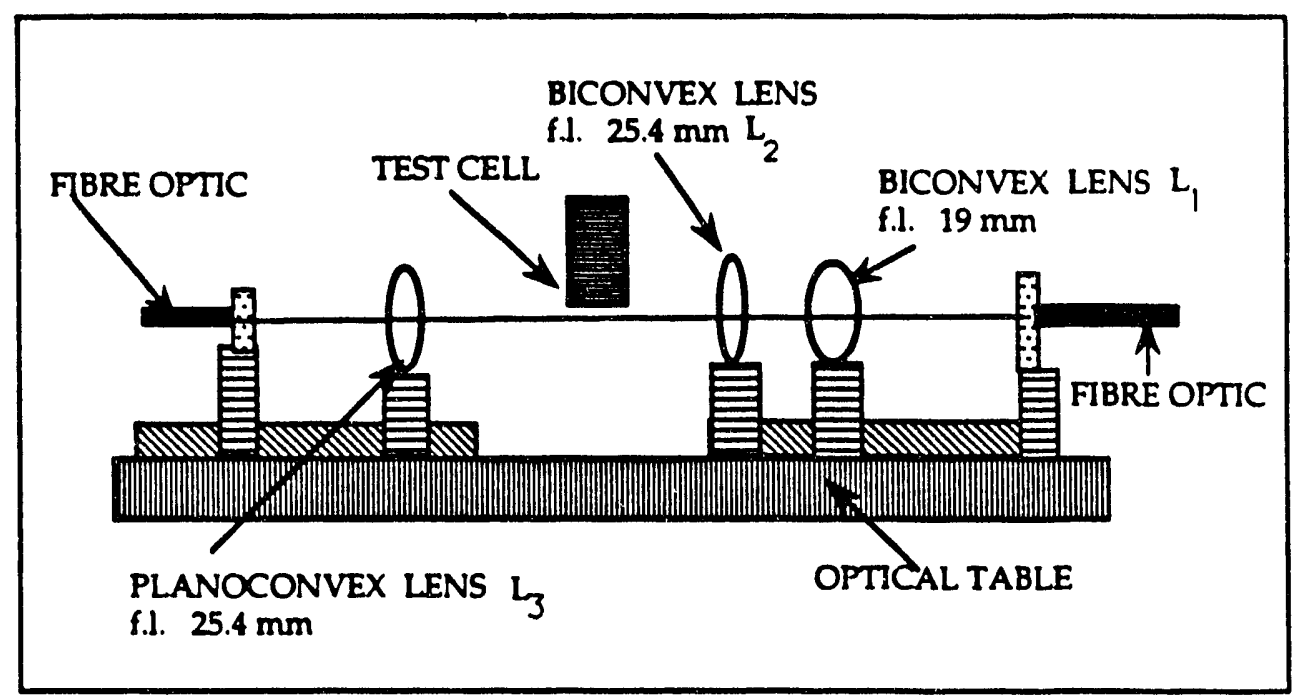

Figure 6 Schematic of Optical System Used for Transmission Measurements 


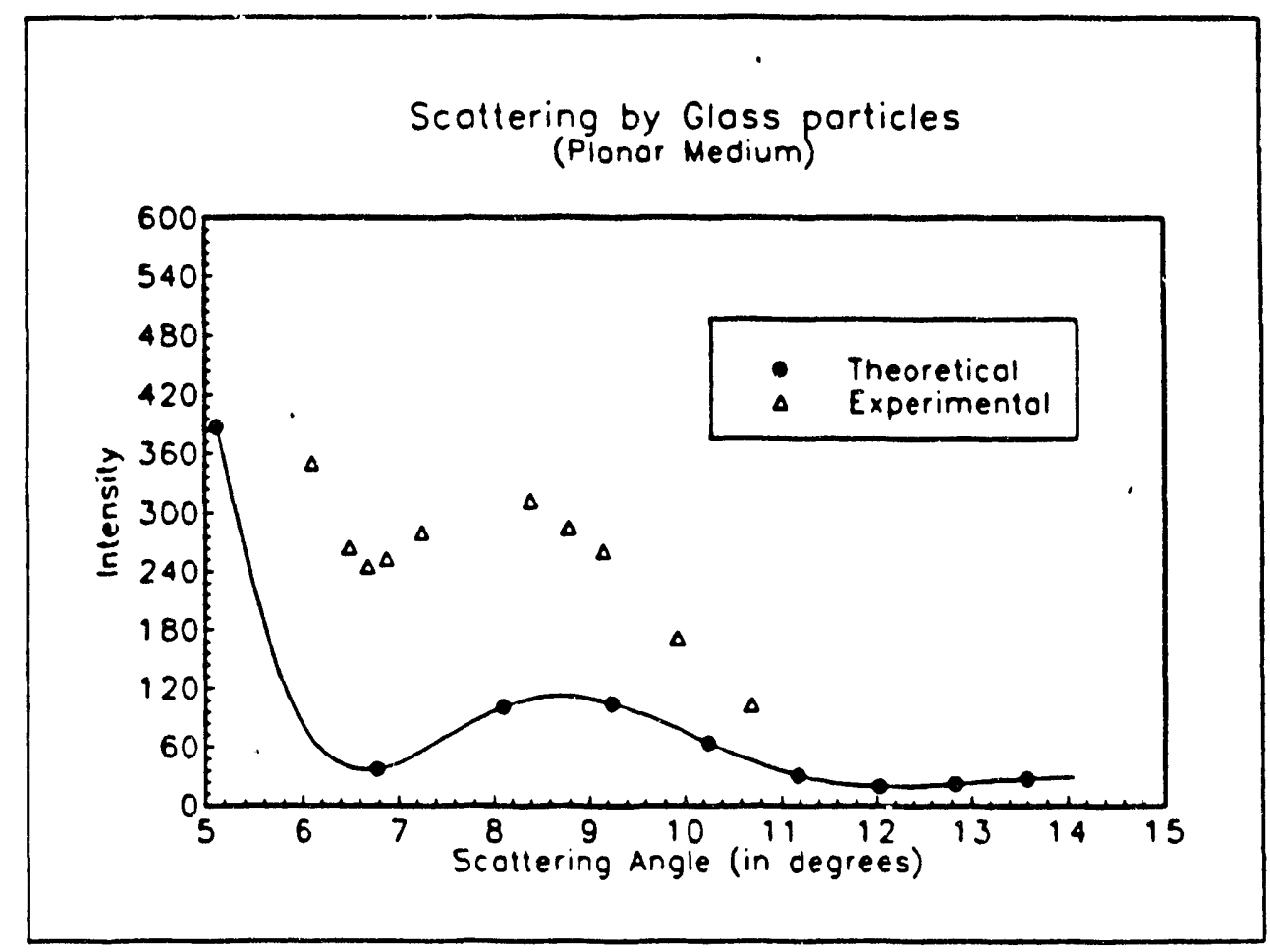

Figure 7 Experimental and Theoretical Angular Distribution of Power for Glass 


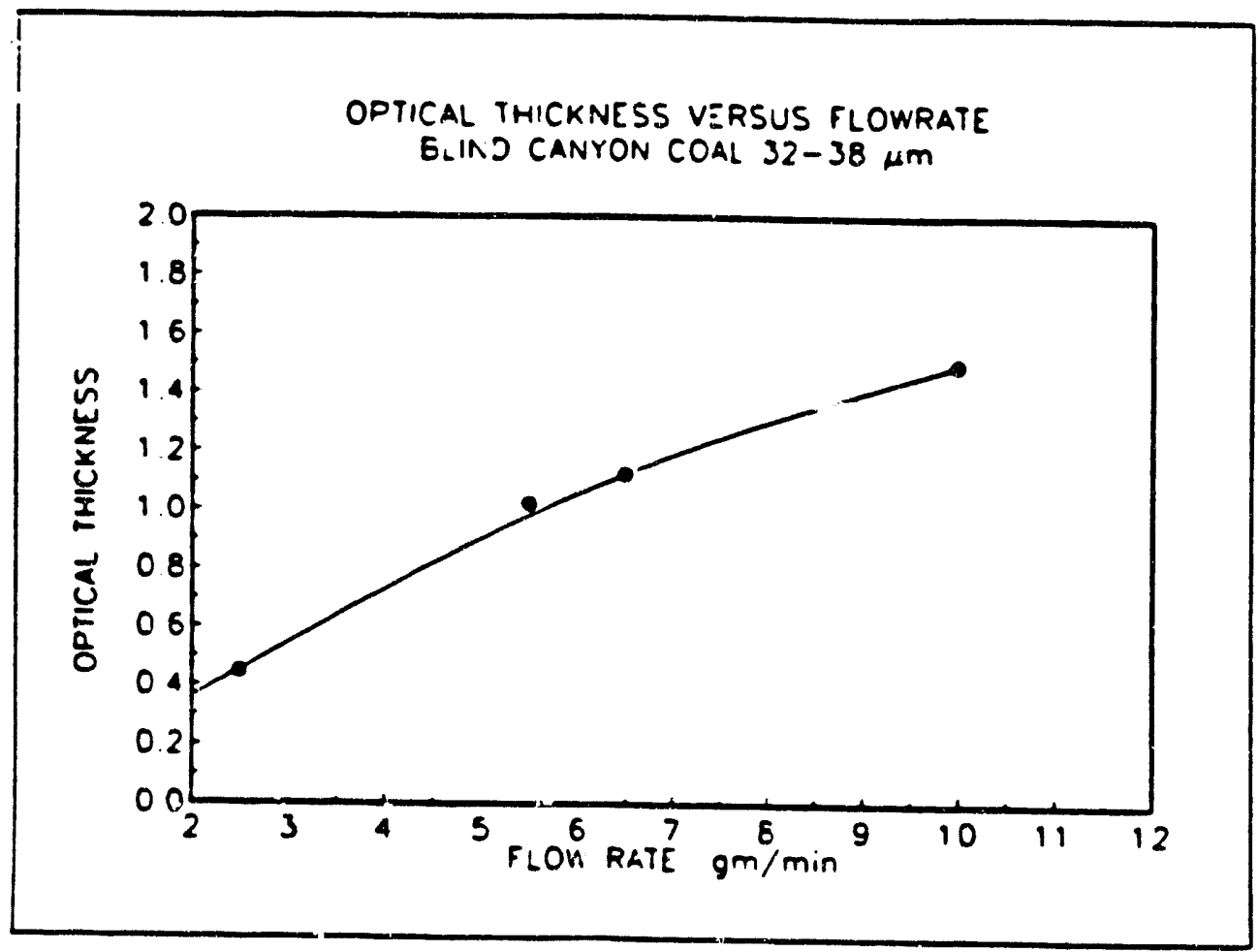

Figure 8 Optical Thickness v 8 Flow-Rate for Blind Canyon Coal $32-38 \mu \mathrm{m}$ 

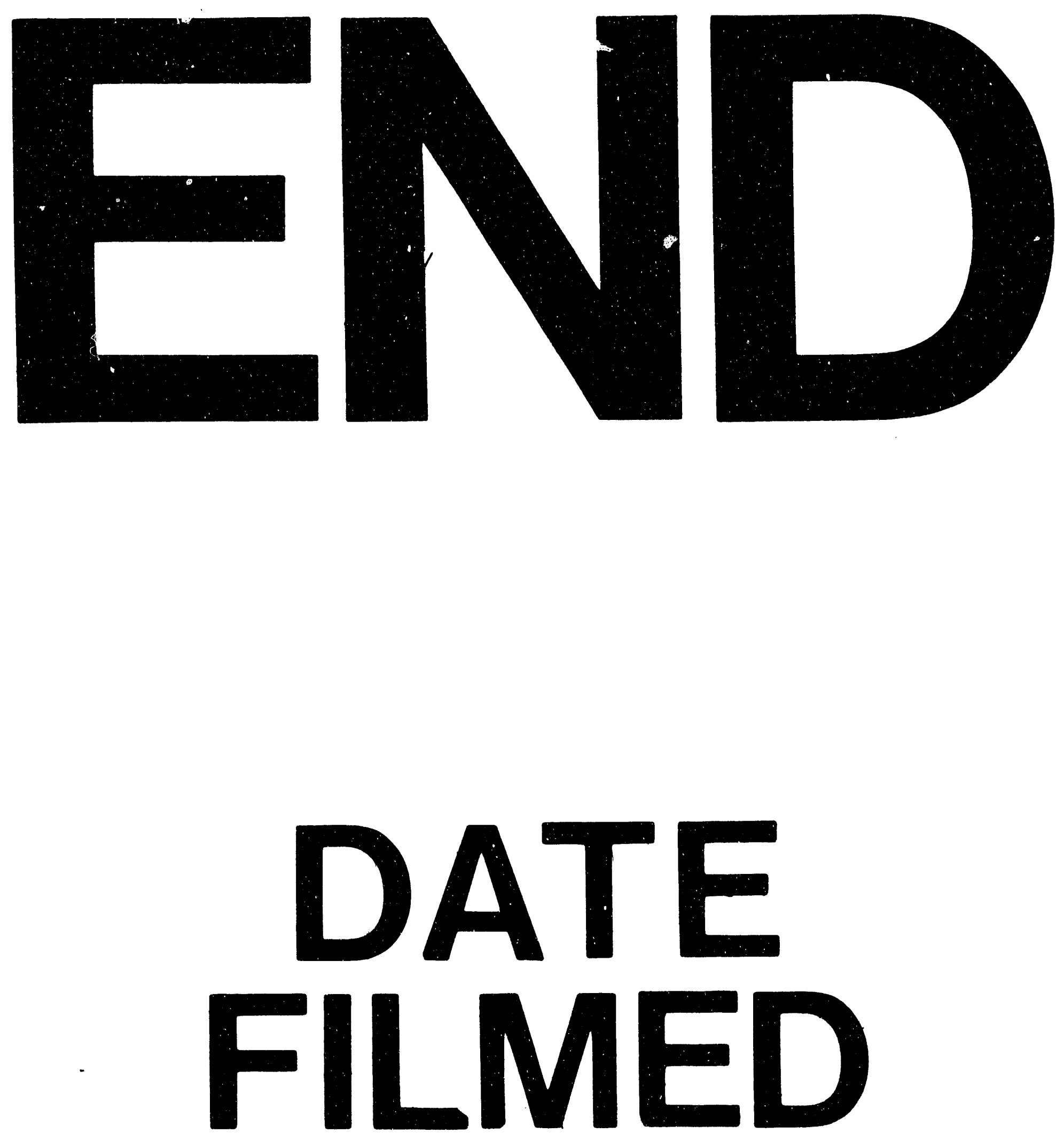

F

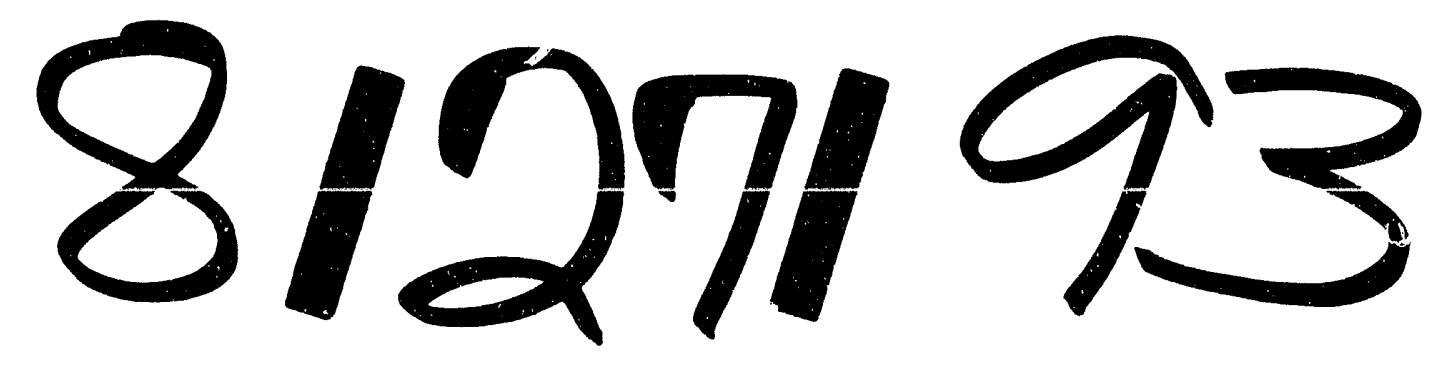


